\title{
CLASSIFICATION OF QUIVER HOPF ALGEBRAS AND POINTED HOPF ALGEBRAS OF TYPE ONE
}

\author{
SHOUCHUAN ZHANG, HUI-XIANG CHEN and YAO-ZHONG ZHANG ${ }^{凶}$
}

(Received 8 March 2012; accepted 27 May 2012; first published online 6 August 2012)

\begin{abstract}
Quiver Hopf algebras are classified by means of ramification systems with irreducible representations. This leads to the classification of Nichols algebras over group algebras and pointed Hopf algebras of type one.
\end{abstract}

2010 Mathematics subject classification: primary 16W30; secondary 16G10.

Keywords and phrases: quivers, Hopf algebras, Hopf bimodules, Nichols algebras.

\section{Introduction}

Hopf algebras have important applications in mathematics and mathematical physics. Indeed, quasitriangular Hopf algebras give rise to braided tensor categories and YangBaxter equations with important applications to integrable systems and statistical mechanics (see, for example, [11] and references therein). Semisimple Hopf algebras and nonsemisimple Hopf algebras are related to conformal field theory (see, for example, [9]).

Classification of Hopf algebras is a main objective in Hopf algebra research. So far, many important results have been obtained in the classification of finite-dimensional pointed Hopf algebras (see, for example, [1-4, 10]). Classification of PM quiver Hopf algebras was completed by means of a ramification system with characters in [20]. The classification of ramification systems with characters over the symmetric group $\mathbb{S}_{n}$ with $n \neq 6$ was obtained in [18]. Irreducible Hopf bimodules over a finite group were described in [8]. They correspond to pairs $\left(O_{s}, \rho\right)$, where $O_{s}$ is a conjugacy class containing $s$ in $\mathrm{G}$ and $\rho$ is an irreducible representation of the centraliser $G^{s}$ in $\mathrm{G}$.

In this paper, quiver Hopf algebras, Nichols algebras over group algebras and pointed Hopf algebras of type one are classified by means of a ramification system with irreducible representations (RSR in short). As examples we classify RSRs over the symmetric group $\mathbb{S}_{n}$ with $n \neq 6$. 
Quivers [6, 7, 14, 19] and tensor algebras of Hopf bimodules [12, 15] have been applied widely in representation theory, Hopf algebras and quantum groups. We use quivers to describe Yetter-Drinfeld $k G$-modules, $k G$-Hopf bimodules, Nichols algebras in the braided tensor category ${ }_{k G}^{k G} \mathcal{Y} D$, pointed Hopf algebras of type one and quiver Hopf algebras.

\section{Preliminaries}

Throughout this paper we assume that $G$ is a finite group and $k$ is a field.

Let $\widehat{G}$ denote the set of all isomorphism classes of irreducible representations of group $G$ and $Z_{s}$ the centraliser of $s$ in $G$. For $h \in G$ and an isomorphism $\phi$ from $G$ to $G^{\prime}$, define a map $\phi_{h}$ from $G$ to $G^{\prime}$ by sending $x$ to $\phi\left(h^{-1} x h\right)$ for any $x \in G$. The dimension of a representation space of representation $\rho$ is denoted by $\operatorname{deg}(\rho)$.

Let $\mathbb{N}$ and $\mathbb{Z}$ denote the sets of all nonnegative integers and all integers, respectively. For a set $X$, we denote by $|X|$ the number of elements in $X$. If $X=\bigoplus_{i \in I} X_{(i)}$ as vector spaces, then we denote by $\iota_{i}$ the natural injection from $X_{(i)}$ to $X$ and by $\pi_{i}$ the corresponding projection from $X$ to $X_{(i)}$. We will use $\mu$ to denote the multiplication of an algebra and use $\Delta$ to denote the comultiplication of a coalgebra. For a (left or right) module and a (left or right) comodule, denote by $\alpha^{-}, \alpha^{+}, \delta^{-}$and $\delta^{+}$the left module, right module, left comodule and right comodule structure maps, respectively. Sweedler's sigma notations for coalgebras and comodules are $\Delta(x)=\sum x_{(1)} \otimes x_{(2)}$, $\delta^{-}(x)=\sum x_{(-1)} \otimes x_{(0)}, \delta^{+}(x)=\sum x_{(0)} \otimes x_{(1)}$.

A quiver $Q=\left(Q_{0}, Q_{1}, s, t\right)$ is an oriented graph, where $Q_{0}$ and $Q_{1}$ are the sets of vertices and arrows, respectively; $s$ and $t$ are two maps from $Q_{1}$ to $Q_{0}$. For any arrow $a \in Q_{1}, s(a)$ and $t(a)$ are called its start vertex and end vertex, respectively, and $a$ is called an arrow from $s(a)$ to $t(a)$. For any $n \geq 0$, an $n$-path or a path of length $n$ in the quiver $Q$ is an ordered sequence of arrows $p=a_{n} a_{n-1} \ldots a_{1}$ with $t\left(a_{i}\right)=s\left(a_{i+1}\right)$ for all $1 \leq i \leq n-1$. Note that a 0 -path is precisely a vertex and a 1 -path is precisely an arrow. In this case, we define $s(p)=s\left(a_{1}\right)$, the start vertex of $p$, and $t(p)=t\left(a_{n}\right)$, the end vertex of $p$. For a 0-path $x$, we have $s(x)=t(x)=x$. Let $Q_{n}$ be the set of $n$-paths. Let ${ }^{y} Q_{n}^{x}$ denote the set of all $n$-paths from $x$ to $y, x, y \in Q_{0}$. That is, ${ }^{y} Q_{n}^{x}=\left\{p \in Q_{n} \mid s(p)=x, t(p)=y\right\}$.

A quiver $Q$ is finite if $Q_{0}$ and $Q_{1}$ are finite sets. A quiver $Q$ is locally finite if ${ }^{y} Q_{1}^{x}$ is a finite set for any $x, y \in Q_{0}$.

Let $G$ be a group. Let $\mathcal{K}(G)$ denote the set of conjugacy classes in $G$. A formal sum $r=\sum_{C \in \mathcal{K}(G)} r_{C} C$ of conjugacy classes of $G$ with cardinal number coefficients is called a ramification (or ramification data) of $G$, that is, for any $C \in \mathcal{K}(G), r_{C}$ is a cardinal number. In particular, a formal sum $r=\sum_{C \in \mathcal{K}(G)} r_{C} C$ of conjugacy classes of $G$ with nonnegative integer coefficients is a ramification of $G$.

For any ramification $r$ and a $C \in \mathcal{K}(G)$, without loss of generality, we can choose a set $I_{C}(r)$ such that its cardinal number is $r_{C}$. Let

$$
\mathcal{K}_{r}(G):=\left\{C \in \mathcal{K}(G) \mid r_{C} \neq 0\right\}=\left\{C \in \mathcal{K}(G) \mid I_{C}(r) \neq \emptyset\right\}
$$


If there exists a ramification $r$ of $G$ such that the cardinal number of ${ }^{y} Q_{1}^{x}$ is equal to $r_{C}$ for any $x, y \in G$ with $x^{-1} y \in C \in \mathcal{K}(G)$, then $Q$ is called a Hopf quiver with respect to the ramification data $r$. In this case, there is a bijection from $I_{C}(r)$ to ${ }^{y} Q_{1}^{x}$, and hence we write ${ }^{y} Q_{1}^{x}=\left\{a_{y, x}^{(i)} \mid i \in I_{C}(r)\right\}$ for any $x, y \in G$ with $x^{-1} y \in C \in \mathcal{K}(G)$. Denote by $(Q, G, r)$ the Hopf quiver of $G$ with respect to $r$.

If $\phi: A \rightarrow A^{\prime}$ is an algebra homomorphism and $\left(M, \alpha^{-}\right)$is a left $A^{\prime}$-module, then $M$ becomes a left $A$-module with the $A$-action given by $a \cdot x=\phi(a) \cdot x$ for any $a \in A$, $x \in M$, called a pullback $A$-module through $\phi$, written as ${ }_{\phi} M$. Dually, if $\phi: C \rightarrow C^{\prime}$ is a coalgebra homomorphism and $\left(M, \delta^{-}\right)$is a left $C$-comodule, then $M$ is a left $C^{\prime}$ comodule with the $C^{\prime}$-comodule structure given by $\delta^{\prime-}:=(\phi \otimes \mathrm{id}) \delta^{-}$, called a push-out $C^{\prime}$-comodule through $\phi$, written as ${ }^{\phi} M$.

If $B$ is a Hopf algebra and $M$ is a $B$-Hopf bimodule, then we say that $(B, M)$ is a Hopf bimodule. For any two Hopf bimodules $(B, M)$ and $\left(B^{\prime}, M^{\prime}\right)$, if $\phi$ is a Hopf algebra homomorphism from $B$ to $B^{\prime}$ and $\psi$ is simultaneously a $B$-bimodule homomorphism from $M$ to ${ }_{\phi} M^{\prime}{ }_{\phi}$ and a $B^{\prime}$-bicomodule homomorphism from ${ }^{\phi} M^{\phi}$ to $M^{\prime}$, then $(\phi, \psi)$ is called a pull-push Hopf bimodule homomorphism. If $\psi$ is a bijection, then we say that $(\phi, \psi)$ is a pull-push Hopf bimodule isomorphism, written as $(B, M) \cong\left(B^{\prime}, M^{\prime}\right)$ as pull-push Hopf bimodules. In particular, if $B=B^{\prime}$ we also write $M \cong M^{\prime}$ as pull-push $B$-Hopf bimodules. Similarly, we say that $(B, M)$ and $(B, X)$ are a Yetter-Drinfeld module and a Yetter-Drinfeld Hopf algebra, respectively, if $M$ is a Yetter-Drinfeld $B$-module and $X$ is a braided Hopf algebra in Yetter-Drinfeld category ${ }_{B}^{B} \mathcal{Y} D$. For any two Yetter-Drinfeld modules $(B, M)$ and $\left(B^{\prime}, M^{\prime}\right)$, if $\phi$ is a Hopf algebra homomorphism from $B$ to $B^{\prime}$, and $\psi$ is simultaneously a left $B$-module homomorphism from $M$ to ${ }_{\phi} M^{\prime}$ and a left $B^{\prime}$-comodule homomorphism from ${ }^{\phi} M$ to $M^{\prime}$, then $(\phi, \psi)$ is called a pull-push Yetter-Drinfeld module homomorphism. For any two Yetter-Drinfeld Hopf algebras $(B, X)$ and $\left(B^{\prime}, X^{\prime}\right)$, if $\phi$ is a Hopf algebra homomorphism from $B$ to $B^{\prime}, \psi$ is simultaneously a left $B$-module homomorphism from $X$ to ${ }_{\phi} X^{\prime}$ and a left $B^{\prime}$-comodule homomorphism from ${ }^{\phi} X$ to $X^{\prime}$, and at the same time $\psi$ is also an algebra and coalgebra homomorphism from $X$ to $X^{\prime}$, then $(\phi, \psi)$ is called a pull-push Yetter-Drinfeld Hopf algebra homomorphism. (See the remark after Theorem 4 in [20].)

Let $A$ be an algebra and $M$ be an $A$-bimodule. Then the tensor algebra $T_{A}(M)$ of $M$ over $A$ is a graded algebra with $T_{A}(M)_{(0)}=A, T_{A}(M)_{(1)}=M$ and $T_{A}(M)_{(n)}=\bigotimes_{A}^{n} M$ for $n>1$. That is,

$$
T_{A}(M)=A \oplus\left(\bigoplus_{n>0} \bigotimes_{A}^{n} M\right)
$$

(see [12]). Let $D$ be another algebra. If $h$ is an algebra map from $A$ to $D$ and $f$ is an $A$-bimodule map from $M$ to $D={ }_{h} D_{h}$, then by the universal property of $T_{A}(M)$ (see [12, Proposition 1.4.1]) there is a unique algebra map $T_{A}(h, f): T_{A}(M) \rightarrow$ $D$ such that $T_{A}(h, f) \iota_{0}=h$ and $T_{A}(h, f) \iota_{1}=f$. We can easily see that $T_{A}(h, f)=$ $h+\sum_{n>0} \mu^{n-1} T_{n}(f)$. For details, the reader is referred to [12, Section 1.4] or [20]. Dually, let $C$ be a coalgebra and let $M$ be a $C$-bicomodule. Then the cotensor 
coalgebra $T_{C}^{c}(M)$ of $M$ over $C$ is a graded coalgebra with $T_{C}^{c}(M)_{(0)}=C, T_{C}^{c}(M)_{(1)}=M$ and $T_{C}^{c}(M)_{(n)}=\square_{C}^{n} M$ for $n>1$. That is,

$$
T_{C}^{c}(M)=C \oplus\left(\bigoplus_{n>0} \prod_{C}^{n} M\right)
$$

(see [12] or [20]). If $B$ is a Hopf algebra and $M$ is a $B$-Hopf bimodule, then both $T_{B}(M)$ and $T_{B}^{c}(M)$ are graded Hopf algebras. Furthermore, the subalgebra generated by $H$ and $M$ in $T_{B}^{c}(M)$, written as $H[M]$, is a Hopf subalgebra of $T_{B}^{c}(M)$ and $H[M]$ is called a Hopf algebra of type one.

\section{Classification of quiver Hopf algebras}

Definition 2.1. We call $(G, r, \vec{\rho}, u)$ a ramification system with irreducible representations (RSR) if $r$ is a ramification of $G$; $u$ is a map from $\mathcal{K}(G)$ to $G$ with $u(C) \in C$ for any $C \in \mathcal{K}(G) ; I_{C}(r, u)$ and $J_{C}(i)$ are sets with $\left|J_{C}(i)\right|=\operatorname{deg}\left(\rho_{C}^{(i)}\right)$ and $I_{C}(r)=\{(i, j) \mid i \in$ $\left.I_{C}(r, u), j \in J_{C}(i)\right\}$ for any $C \in \mathcal{K}_{r}(G), i \in I_{C}(r, u)$; and

$$
\vec{\rho}=\left\{\rho_{C}^{(i)}\right\}_{i \in I_{C}(r, u), C \in \mathcal{K}_{r}(G)} \in \prod_{C \in \mathcal{K}_{r}(G)}\left(\widehat{Z_{u(C)}}\right)^{\left|I_{C}(r, u)\right|}
$$

with $\rho_{C}^{(i)} \in \widehat{Z_{u(C)}}$ for any $i \in I_{C}(r, u), C \in \mathcal{K}_{r}(G)$. Also $\operatorname{RSR}(G, r, \vec{\rho}, u)$ and $\operatorname{RSR}\left(G^{\prime}, r^{\prime}, \overrightarrow{\rho^{\prime}}, u^{\prime}\right)$ are said to be isomorphic if the following conditions are satisfied.

- There exists a group isomorphism $\phi: G \rightarrow G^{\prime}$.

- For any $C \in \mathcal{K}(G)$, there exists an element $h_{C} \in G$ such that $\phi\left(h_{C}^{-1} u(C) h_{C}\right)=$ $u^{\prime}(\phi(C))$.

- $\quad$ For any $C \in \mathcal{K}_{r}(G)$, there exists a bijective map $\phi_{C}: I_{C}(r, u) \rightarrow I_{\phi(C)}\left(r^{\prime}, u^{\prime}\right)$ such that $\rho_{C}^{(i)} \cong \rho_{\phi(C)}^{\prime\left(\phi_{C}(i)\right)} \phi_{h_{c}}$ as representations of $k Z_{u(C)}$ for all $i \in I_{C}(r, u)$, where $\phi_{h_{C}}(h)=\phi\left(h_{C}^{-1} h h_{C}\right)$ for any $h \in G$.

Remark 2.2. Assume that $G=G^{\prime}, r=r^{\prime}, u(C)=u^{\prime}(C)$ and $I_{C}(r, u)=I_{C}\left(r^{\prime}, u^{\prime}\right)$ for any $C \in \mathcal{K}_{r}(G)$. If there is a permutation $\phi_{C}$ on $I_{C}(r, u)$ for any $C \in \mathcal{K}_{r}(G)$ such that $\rho_{C}^{\prime\left(\phi_{C}(i)\right)} \cong \rho_{C}^{(i)}$ for all $i \in I_{C}(r, u)$, then obviously $\operatorname{RSR}(G, r, \vec{\rho}, u) \cong \operatorname{RSR}\left(G, r, \overrightarrow{\rho^{\prime}}, u\right)$.

Example 2.3. Assume that $k$ is a complex field and $G=\mathbb{S}_{3}$. Then there are three elements in $\mathcal{K}(G)$, which are $\{(1)\},\{(12),(13),(23)\},\{(123),(132)\}$, and there are three nonisomorphic irreducible representations, namely two one-dimensional irreducible representations, $\epsilon$ and sgn, and one two-dimensional irreducible representation, $\rho$. Obviously $Z_{u(\{1\})}=G$. The set $\{\operatorname{RSR}(G, r, \vec{\rho}, u) \mid \vec{\rho}=\rho,(\operatorname{sgn}, \operatorname{sgn}),(\epsilon, \operatorname{sgn}),(\epsilon, \epsilon)\}$ gives all the representatives of isomorphic classes of RSRs with $r=r_{C} r$ and $C=\{(1)\}$. Furthermore, when $\vec{\rho}=\rho$, we can set $I_{C}(r, u)=\{1\}$ and $J_{C}(1)=\{1\}$. In this case $\rho_{C}^{(1)}=\rho$. When $\vec{\rho}=(\epsilon$, sgn $)$, we can set $I_{C}(r, u)=\{1,2\}$ and $J_{C}(1)=J_{C}(2)=\{1\}$. In this case $\rho_{C}^{(1)}=\epsilon, \rho_{C}^{(2)}=\operatorname{sgn}$. 
Let

$$
G=\bigcup_{\theta \in \Theta_{C}} Z_{u(C)} g_{\theta},
$$

where $\Theta_{C}$ is an index set, be a coset decomposition of $Z_{u(C)}$ in $G$. It is easy to check that $\left|\Theta_{C}\right|=|C|$. We always assume that the representative element of the coset $Z_{u(C)}$ is the identity 1 of $G$. For any $h \in G$ and $\theta \in \Theta_{C}$, there exist unique $h^{\prime} \in Z_{u(C)}$ and $\theta^{\prime} \in \Theta_{C}$ such that $g_{\theta} h=h^{\prime} g_{\theta^{\prime}}$. Let $\zeta_{\theta}(h)=h^{\prime}$. Then

$$
g_{\theta} h=\zeta_{\theta}(h) g_{\theta^{\prime}}
$$

Let $Q=\left(G, Q_{1}, s, t\right)$ be a quiver of a group $G$. Then $k Q_{1}$ becomes a $k G$-bicomodule under the natural comodule structures:

$$
\delta^{-}(a)=t(a) \otimes a, \quad \delta^{+}(a)=a \otimes s(a), \quad a \in Q_{1},
$$

called an arrow comodule, written as $k Q_{1}^{c}$. In this case, the path coalgebra $k Q^{c}$ is exactly isomorphic to the cotensor coalgebra $T_{k G}^{c}\left(k Q_{1}^{c}\right)$ over $k G$ in a natural way (see $[6,7])$. We will set $k Q^{c}=T_{k G}^{c}\left(k Q_{1}^{c}\right)$ in the following. Moreover, when $G$ is finite, $k Q_{1}$ becomes a $(k G)^{*}$-bimodule with the module structures defined by

$$
p \cdot a:=\langle p, t(a)\rangle a, \quad a \cdot p:=\langle p, s(a)\rangle a, \quad p \in(k G)^{*}, a \in Q_{1},
$$

written as $k Q_{1}^{a}$, called an arrow module. Therefore, we have a tensor algebra $T_{(k G)^{*}}\left(k Q_{1}^{a}\right)$. Note that the tensor algebra $T_{(k G)^{*}}\left(k Q_{1}^{a}\right)$ of $k Q_{1}^{a}$ over $(k G)^{*}$ is exactly isomorphic to the path algebra $k Q^{a}$. We will set $k Q^{a}=T_{(k G)^{*}}\left(k Q_{1}^{a}\right)$ in the following.

Proposition 2.4. If $N$ is a $k G$-Hopf bimodule, then there exist a Hopf quiver $(Q, G, r)$, an $\operatorname{RSR}(G, r, \vec{\rho}, u)$ and a $k G$-Hopf bimodule $\left(k Q_{1}^{c}, \alpha^{-}, \alpha^{+}\right)$with

$$
\alpha^{-}\left(h \otimes a_{y, x}^{(i, j)}\right):=h \cdot a_{y, x}^{(i, j)}=a_{h y, h x}^{(i, j)}, \quad \alpha^{+}\left(a_{y, x}^{(i, j)} \otimes h\right):=a_{y, x}^{(i, j)} \cdot h=\sum_{s \in J_{C}(i)} k_{C, h}^{(i, j, s)} a_{y h, x h}^{(i, s)}
$$

for some $k_{C, h}^{(i, j, s)} \in k$ such that $N \cong\left(k Q_{1}^{c}, \alpha^{-}, \alpha^{+}\right)$as $k G$-Hopf bimodules, where $x, y, h \in$ $G$ with $x^{-1} y=g_{\theta}^{-1} u(C) g_{\theta}, \zeta_{\theta}$ is given by [20, (0.3)], and

$$
C \in \mathcal{K}_{r}(G), \quad i \in I_{C}(r, u), \quad j \in J_{C}(i), \quad x_{C}^{(i, j)} \cdot \zeta_{\theta}(h)=\sum_{s \in J_{C}(i)} k_{C, h}^{(i, j, s)} x_{C}^{(i, s)} .
$$

Proof. Since $N$ is a $k G$-Hopf bimodule, there exists an object $\prod_{C \in \mathcal{K}(G)} M(C)$ in $\prod_{C \in \mathcal{K}(G)} \mathcal{M}_{k Z_{u(C)}}$ such that $M(C)$ is a $k Z_{u(C)}$-module for any $C \in \mathcal{K}(G)$ and



as $k G$-Hopf bimodules by [6] or [20, Theorem 1]. Let $r=\sum_{C \in \mathcal{K}(G)} r_{C} C$ with $r_{C}=$ $\operatorname{dim} M(C)$ for any $C \in \mathcal{K}(G)$. Notice that $\operatorname{dim} M(C)$ denotes the cardinal number 
of a basis of $M(C)$ when $M(C)$ is infinite-dimensional. Since $M(C)$ is a $k Z_{u(C)^{-}}$ module and $k Z_{u(C)}$ is semisimple, there exists a family of irreducible representations $\left\{\left(X_{C}^{(i)}, \rho_{C}^{(i)}\right) \mid i \in I_{C}(r, u)\right\}$ such that

$$
M(C)=\bigoplus_{i \in I_{C}(r, u)}\left(X_{C}^{(i)}, \rho_{C}^{(i)}\right) .
$$

Let $\left\{x_{C}^{(i, j)} \mid j \in J_{C}(i)\right\}$ be a $k$-basis of $X_{C}^{(i)}$ for any $i \in I_{C}(r, u)$. Then for any $h \in G$ there are some $k_{C, h}^{(i, j, s)} \in k$ such that

$$
x_{C}^{(i, j)} \cdot \zeta_{\theta}(h)=\sum_{s \in J_{C}(i)} k_{C, h}^{(i, j, s)} x_{C}^{(i, s)}
$$

for all $i \in I_{C}(r, u)$ and $j \in J_{C}(i)$ since $x_{C}^{(i, j)} \cdot \zeta_{\theta}(h) \in X_{C}^{(i)}$.

It remains to show that $\left(k Q_{1}^{c}, \alpha^{-}, \alpha^{+}\right)$is isomorphic to

$$
\bigoplus_{y=x g_{\theta}^{-1} u(C) g_{\theta}, x, y \in G} x \otimes M(C) \bigotimes_{k Z_{u(C)}} g_{\theta}
$$

as $k G$-Hopf bimodules. Observe that there is a canonical $k G$-bicomodule isomorphism

$$
\varphi: k Q_{1} \rightarrow \bigoplus_{y=x g_{\theta}^{-1} u(C) g_{\theta}, x, y \in G} x \otimes M(C) \bigotimes_{k Z_{u(C)}} g_{\theta}
$$

given by

$$
\varphi\left(a_{y, x}^{(i, j)}\right)=x \otimes x_{C}^{(i, j)} \bigotimes_{k Z_{u(C)}} g_{\theta}
$$

where $x, y \in G$ with $x^{-1} y=g_{\theta}^{-1} u(C) g_{\theta}, C \in \mathcal{K}_{r}(G), i \in I_{C}(r, u)$ and $j \in J_{C}(r)$. Then

$$
\begin{aligned}
\varphi\left(\alpha^{-}\left(h \otimes a_{y, x}^{(i, j)}\right)\right) & =\varphi\left(a_{h y, h x}^{(i, j)}\right)=h x \otimes x_{C}^{(i, j)} \bigotimes_{k Z_{u(C)}} g_{\theta} \\
& =h \cdot\left(x \otimes x_{C}^{(i, j)} \bigotimes_{k Z_{u(C)}} g_{\theta}\right) \quad(\operatorname{see}[20,(1.2)]) \\
& =h \cdot \varphi\left(a_{y, x}^{(i, j)}\right) .
\end{aligned}
$$

Thus $\varphi$ is a left $k G$-module isomorphism. Moreover,

$$
\begin{aligned}
\alpha^{+}\left(\varphi\left(a_{y, x}^{(i, j)}\right) \otimes h\right) & =x h \otimes x_{C}^{(i, j)} \cdot \zeta_{\theta}(h) \otimes g_{\theta^{\prime}} \\
& =x h \otimes\left(\sum_{s \in J_{C}(i)} k_{C, h}^{(i, j, s)} x_{C}^{(i, s)}\right) \otimes g_{\theta^{\prime}} \\
& =\varphi\left(\sum_{s \in J_{C}(i)} k_{C, h}^{(i, j, s)} a_{y h, x h}^{(i, s)}\right) \\
& =\varphi\left(\alpha^{+}\left(a_{y, x}^{(i, j)} \otimes h\right)\right) \quad(\text { by }(2.2)) .
\end{aligned}
$$

Consequently, $\varphi$ is a $k G$-Hopf bimodule isomorphism. 
Let $\left(k Q_{1}^{c}, G, r, \vec{\rho}, u\right)$ denote the $k G$-Hopf bimodule $\left(k Q_{1}^{c}, \alpha^{-}, \alpha^{+}\right)$given in Proposition 2.4. Furthermore, if $\left(k Q_{1}^{c}, k Q_{1}^{a}\right)$ is an arrow dual pairing, that is, $k Q_{1}^{c}$ is isomorphic to the dual of $k Q_{1}^{a}$ as $k G$-Hopf bimodules or $k Q_{1}^{a}$ is isomorphic to the dual of $k Q_{1}^{c}$ as $(k G)^{*}$-Hopf bimodules under the isomorphisms in [20, Lemma 1.7] (see the argument before [20, Definition 1.8]), then we denote the $(k G)^{*}$-Hopf bimodule $k Q_{1}^{a}$ by $\left(k Q_{1}^{a}, G, r, \vec{\rho}, u\right)$. We obtain six quiver Hopf algebras $k Q^{c}(G, r, \vec{\rho}, u), k Q^{s}(G, r, \vec{\rho}, u)$, $k G\left[k Q_{1}^{c}, G, r, \vec{\rho}, u\right], k Q^{a}(G, r, \vec{\rho}, u), k Q^{s c}(G, r, \vec{\rho}, u),(k G)^{*}\left[k Q_{1}^{a}, G, r, \vec{\rho}, u\right]$, called the quiver Hopf algebras determined by $\operatorname{RSR}(G, r, \vec{\rho}, u)$.

From Proposition 2.4, it seems that the right $k G$-action on $\left(k Q_{1}^{c}, G, r, \vec{\rho}, u\right)$ depends on the choice of the set $\left\{g_{\theta} \mid \theta \in \Theta_{C}\right\}$ of coset representatives of $Z_{u(C)}$ in $G$ (see (2.1) above or $[20,(0.1)])$. The following lemma shows that $\left(k Q_{1}^{c}, G, r, \vec{\rho}, u\right)$ is, in fact, independent of the choice of the coset representative set $\left\{g_{\theta} \mid \theta \in \Theta_{C}\right\}$, up to $k G$ Hopf bimodule isomorphisms. For the time being, we write $\left(k Q_{1}^{c}, G, r, \vec{\rho}, u\right)=$ $\left(k Q_{1}^{c}, G, r, \vec{\rho}, u,\left\{g_{\theta}\right\}\right)$, as given before. Now let $\left\{h_{\theta} \in G \mid \theta \in \Theta_{C}\right\}$ be another coset representative set of $Z_{u(C)}$ in $G$ for any $C \in \mathcal{K}(G)$. That is,

$$
G=\bigcup_{\theta \in \Theta_{C}} Z_{u(C)} h_{\theta} .
$$

Lemma 2.5. With the above notation, $\left(k Q_{1}^{c}, G, r, \vec{\rho}, u,\left\{g_{\theta}\right\}\right)$ and $\left(k Q_{1}^{c}, G, r, \vec{\rho}, u,\left\{h_{\theta}\right\}\right)$ are isomorphic $k G$-Hopf bimodules.

Proof. We may assume that $Z_{u(C)} h_{\theta}=Z_{u(C)} g_{\theta}$ for any $C \in \mathcal{K}(G)$ and $\theta \in \Theta_{C}$. Then $g_{\theta} h_{\theta}^{-1} \in Z_{u(C)}$. Now let $x, y, h \in G$ with $x^{-1} y=g_{\theta}^{-1} u(C) g_{\theta}$. Then

$$
x^{-1} y=h_{\theta}^{-1}\left(g_{\theta} h_{\theta}^{-1}\right)^{-1} u(C)\left(g_{\theta} h_{\theta}^{-1}\right) h_{\theta}=h_{\theta}^{-1} u(C) h_{\theta}
$$

and

$$
h_{\theta} h=\left(h_{\theta} g_{\theta}^{-1}\right) g_{\theta} h=\left(h_{\theta} g_{\theta}^{-1}\right) \zeta_{\theta}(h) g_{\theta^{\prime}}=\left(h_{\theta} g_{\theta}^{-1}\right) \zeta_{\theta}(h)\left(g_{\theta^{\prime}} h_{\theta^{\prime}}^{-1}\right) h_{\theta^{\prime}},
$$

where $g_{\theta} h=\zeta_{\theta}(h) g_{\theta^{\prime}}$.

For any $C \in \mathcal{K}(G)$, let $M(C)$ be a right $k Z_{u(C)}$-module. Let

$$
N:=\bigoplus_{y=x g_{\theta}^{-1} u(C) g_{\theta}, x, y \in G} x \otimes M(C) \bigotimes_{k Z_{u(C)}} g_{\theta}
$$

and

$$
M:=\bigoplus_{y=x h_{\theta}^{-1} u(C) h_{\theta}, x, y \in G} x \otimes M(C) \bigotimes_{k Z_{u(C)}} h_{\theta}
$$

be two $k G$-Hopf bimodules. It is sufficient to show $N \cong M$ as $k G$-Hopf bimodules by the proof of Proposition 2.4.

Considering

$$
x \otimes w \bigotimes_{k Z_{u(C)}} g_{\theta}=x \otimes w \cdot g_{\theta} h_{\theta}^{-1} \bigotimes_{k Z_{u(C)}} h_{\theta},
$$


we have that $f: N \rightarrow M$ given by

$$
f\left(x \otimes w \bigotimes_{k Z_{u(C)}} g_{\theta}\right)=x \otimes w \cdot g_{\theta} h_{\theta}^{-1} \bigotimes_{k Z_{u(C)}} h_{\theta}
$$

for any $w \in M(C)$, any $x, y \in G$ with $x^{-1} y=g_{\theta}^{-1} u(C) g_{\theta}, C \in \mathcal{K}_{r}(G)$ and $i \in I_{C}(r, u)$, is a $k$-linear isomorphism. It is clear that $f$ is a $k G$-bicomodule isomorphism and a left $k G$-module isomorphism from $N$ to $M$. Moreover,

$$
\begin{aligned}
(f(x & \left.\left.\otimes x_{C}^{(i, j)} \bigotimes_{k Z_{u(C)}} g_{\theta}\right)\right) \cdot h \\
& =\left(x \otimes\left(x_{C}^{(i, j)}\right) \rho_{C}^{(i)}\left(g_{\theta} h_{\theta}^{-1}\right) \bigotimes_{k Z_{u(C)}} h_{\theta}\right) \cdot h \\
& =x h \otimes\left(x_{C}^{(i, j)}\right) \rho_{C}^{(i)}\left(\zeta_{\theta}(h)\right) \rho_{C}^{(i)}\left(g_{\theta^{\prime}} h_{\theta^{\prime}}^{-1}\right) \bigotimes_{k Z_{u(C)}} h_{\theta^{\prime}} \quad(\text { by }(2.3)) \\
& =f\left(\left(x \otimes x_{C}^{(i, j)} \bigotimes_{k Z_{u(C)}} g_{\theta}\right) \cdot h\right),
\end{aligned}
$$

for any $x, y, h \in G, i \in I_{C}(r, u), j \in J_{C}(i), C \in \mathcal{K}_{r}(G)$ with $x^{-1} y=g_{\theta}^{-1} u(C) g_{\theta}$. Thus $f$ is a right $k G$-module homomorphism.

We now state one of our main results, which classifies the quiver Hopf algebras.

Theorem 2.6. Let $(G, r, \vec{\rho}, u)$ and $\left(G^{\prime}, r^{\prime}, \overrightarrow{\rho^{\prime}}, u^{\prime}\right)$ be two RSRs. Then the following statements are equivalent.

(i) $\operatorname{RSR}(G, r, \vec{\rho}, u) \cong \operatorname{RSR}\left(G^{\prime}, r^{\prime}, \overrightarrow{\rho^{\prime}}, u^{\prime}\right)$.

(ii) There exists a Hopf algebra isomorphism $\phi: k G \rightarrow k G^{\prime}$ such that

$$
\left(k Q_{1}^{c}, G, r, \vec{\rho}, u\right) \cong \phi_{\phi}^{\phi^{-1}}\left(\left(k Q_{1}^{\prime c}, G^{\prime}, r^{\prime}, \vec{\rho}^{\prime}, u^{\prime}\right)\right)_{\phi}^{\phi^{-1}}
$$

as $k G$-Hopf bimodules.

(iii) $k Q^{c}(G, r, \vec{\rho}, u) \cong k Q^{\prime c}(G, r, \vec{\rho}, u)$.

(iv) $k Q^{s}(G, r, \vec{\rho}, u) \cong k Q^{\prime s}\left(G^{\prime}, r^{\prime}, \vec{\rho}^{\prime}, u^{\prime}\right)$.

(v) $k G\left[k Q_{1}^{c}, G, r, \vec{\rho}, u\right] \cong k G^{\prime}\left[k Q_{1}^{\prime c}, G^{\prime}, r^{\prime}, \vec{\rho}^{\prime}, u^{\prime}\right]$.

Furthermore, if $Q$ is finite, then the above are equivalent to the following.

(vi) $k Q^{a}(G, r, \vec{\rho}, u) \cong k Q^{\prime a}\left(G^{\prime}, r^{\prime}, \vec{\rho}^{\prime}, u^{\prime}\right)$.

(vii) $k Q^{s c}(G, r, \vec{\rho}, u) \cong k Q^{\prime s c}\left(G^{\prime}, r^{\prime}, \vec{\rho}^{\prime}, u^{\prime}\right)$.

(viii) $(k G)^{*}\left[k Q_{1}^{a}, G, r, \vec{\rho}, u\right] \cong\left(k G^{\prime}\right)^{*}\left[k Q_{1}^{\prime a}, G^{\prime}, r^{\prime}, \vec{\rho}^{\prime}, u^{\prime}\right]$.

Proof. By [20, Lemmas 1.5 and 1.6], we only have to prove (i) $\Leftrightarrow$ (ii).

(i) $\Rightarrow$ (ii). Assume that $\operatorname{RSR}(G, r, \vec{\rho}, u) \cong \operatorname{RSR}\left(G^{\prime}, r^{\prime}, \overrightarrow{\rho^{\prime}}, u^{\prime}\right)$. Let $\left(X_{C}^{(i)}, \rho_{C}^{(i)}\right)$ and $\left(X_{C^{\prime}}^{\prime\left(i^{\prime}\right)}, \rho_{C^{\prime}}^{\prime\left(i^{\prime}\right)}\right)$ be irreducible representations over $Z_{u(C)}$ and $Z_{u^{\prime}\left(C^{\prime}\right)}$, respectively. 
Then there exist a group isomorphism $\phi: G \rightarrow G^{\prime}$, an element $h_{C} \in G$ such that $\phi\left(h_{C}^{-1} u(C) h_{C}\right)=u^{\prime}(\phi(C))$ for any $C \in \mathcal{K}(G)$ and a bijective map $\phi_{C}: I_{C}(r, u) \rightarrow$ $I_{\phi(C)}\left(r^{\prime}, u^{\prime}\right)$ such that $\left(X_{C}^{(i)}, \rho_{C}^{(i)}\right) \stackrel{\xi_{\mathcal{C}}^{(i)}}{\cong}\left(X_{\phi(C)}^{\prime\left(\phi_{C}(i)\right)}, \rho_{\phi(C)}^{\left(\phi_{C}(i)\right)} \phi_{h_{c}}\right)$ as right $k Z_{u(C)}$-modules for all $i \in I_{C}(r, u)$.

Now let

$$
G=\bigcup_{\theta \in \Theta_{C}} Z_{u(C)} g_{\theta}
$$

as in (2.1) or [20, (0.1)] for any $C \in \mathcal{K}(G)$. Let $x, y, h \in G$ with $x^{-1} y=g_{\theta}^{-1} u(C) g_{\theta}$, $C \in \mathcal{K}_{r}(G)$ and $\theta \in \Theta_{C}$. Assume that

$$
g_{\theta} h_{C}^{-1}=\zeta_{\theta}\left(h_{C}^{-1}\right) g_{\eta}, \quad g_{\theta} h=\zeta_{\theta}(h) g_{\theta^{\prime}}, \quad g_{\eta}\left(h_{C} h h_{C}^{-1}\right)=\zeta_{\eta}\left(h_{C} h h_{C}^{-1}\right) g_{\eta^{\prime}}
$$

and

$$
g_{\theta^{\prime}} h_{C}^{-1}=\zeta_{\theta^{\prime}}\left(h_{C}^{-1}\right) g_{\theta^{\prime \prime}}
$$

with $\zeta_{\theta}\left(h_{C}^{-1}\right), \zeta_{\theta}(h), \zeta_{\eta}\left(h_{C} h h_{C}^{-1}\right), \zeta_{\theta^{\prime}}\left(h_{C}^{-1}\right) \in Z_{u(C)}$ and $\eta, \theta^{\prime}, \eta^{\prime}, \theta^{\prime \prime} \in \Theta_{C}$. Then

$$
g_{\theta} h h_{C}^{-1}=\zeta_{\theta}(h) g_{\theta^{\prime}} h_{C}^{-1}=\zeta_{\theta}(h) \zeta_{\theta^{\prime}}\left(h_{C}^{-1}\right) g_{\theta^{\prime \prime}}
$$

and

$$
g_{\theta} h h_{C}^{-1}=\left(g_{\theta} h_{C}^{-1}\right)\left(h_{C} h h_{C}^{-1}\right)=\zeta_{\theta}\left(h_{C}^{-1}\right) g_{\eta}\left(h_{C} h h_{C}^{-1}\right)=\zeta_{\theta}\left(h_{C}^{-1}\right) \zeta_{\eta}\left(h_{C} h h_{C}^{-1}\right) g_{\eta^{\prime}} .
$$

It follows that

$$
\theta^{\prime \prime}=\eta^{\prime} \quad \text { and } \quad \zeta_{\theta}(h) \zeta_{\theta^{\prime}}\left(h_{C}^{-1}\right)=\zeta_{\theta}\left(h_{C}^{-1}\right) \zeta_{\eta}\left(h_{C} h h_{C}^{-1}\right) .
$$

Moreover,

$$
(x h)^{-1}(y h)=h^{-1} g_{\theta}^{-1} u(C) g_{\theta} h=g_{\theta^{\prime}}^{-1} u(C) g_{\theta^{\prime}}
$$

and

$$
g_{\theta}=g_{\theta} h_{C}^{-1} h_{C}=\zeta_{\theta}\left(h_{C}^{-1}\right) g_{\eta} h_{C} .
$$

Thus

$$
\begin{aligned}
\phi(x)^{-1} \phi(y) & =\phi\left(x^{-1} y\right)=\phi\left(g_{\theta}^{-1} u(C) g_{\theta}\right)=\phi\left(h_{C}^{-1} g_{\eta}^{-1} u(C) g_{\eta} h_{C}\right) \\
& =\phi\left(h_{C}^{-1} g_{\eta}^{-1} h_{C}\right) \phi\left(h_{C}^{-1} u(C) h_{C}\right) \phi\left(h_{C}^{-1} g_{\eta} h_{C}\right) \\
& =\phi\left(h_{C}^{-1} g_{\eta} h_{C}\right)^{-1} u^{\prime}(\phi(C)) \phi\left(h_{C}^{-1} g_{\eta} h_{C}\right) .
\end{aligned}
$$

Also

$$
\begin{aligned}
\phi\left(h_{C}^{-1} g_{\eta} h_{C}\right) \phi(h) & =\phi\left(h_{C}^{-1} g_{\eta} h_{C} h h_{C}^{-1} h_{C}\right) \\
& =\phi\left(h_{C}^{-1} \zeta_{\eta}\left(h_{C} h h_{C}^{-1}\right) g_{\eta^{\prime}} h_{C}\right) \\
& =\phi\left(h_{C}^{-1} \zeta_{\eta}\left(h_{C} h h_{C}^{-1}\right) h_{C}\right) \phi\left(h_{C}^{-1} g_{\eta^{\prime}} h_{C}\right) .
\end{aligned}
$$


Since

$$
\begin{aligned}
\phi_{h_{C}}\left(g_{\eta^{\prime}}\right) \phi(x h)^{-1} \phi(y h) & =\phi\left(h_{C}^{-1}\left(\zeta_{\eta}\left(h_{C} h h_{C}^{-1}\right)\right)^{-1}\left(\zeta_{\theta}\left(h_{C}^{-1}\right)\right)^{-1} u(C) g_{\theta} h\right) \quad(\text { by 2.4) } \\
& =u^{\prime}(\phi(C)) \phi_{h_{C}}\left(g_{\eta^{\prime}}\right) \quad(\text { by 2.4), }
\end{aligned}
$$

it follows that

$$
\phi(x h)^{-1} \phi(y h)=\phi_{h_{C}}\left(g_{\eta^{\prime}}\right)^{-1} u^{\prime}(\phi(C)) \phi_{h_{C}}\left(g_{\eta^{\prime}}\right)
$$

It is clear that

$$
G^{\prime}=\bigcup_{\theta \in \Theta_{C}} Z_{u^{\prime}(\phi(C))}\left(\phi\left(h_{C}^{-1} g_{\theta} h_{C}\right)\right)
$$

is a coset decomposition of $Z_{u^{\prime}(\phi(C))}$ in $G^{\prime}$ for any $\phi(C) \in \mathcal{K}\left(G^{\prime}\right)$.

Let

$$
N:=\bigoplus_{y=x g_{\theta}^{-1} u(C) g_{\theta}, x, y \in G} x \otimes N(C) \bigotimes_{k Z_{u(C)}} g_{\theta}
$$

and

$$
M:=\bigoplus_{\phi(y)=\phi(x) \phi_{h_{C}}\left(g_{\eta}^{-1}\right) u^{\prime}\left(\phi(C) \phi_{h_{C}}\left(g_{\eta}\right), x, y \in G\right.} \phi(x) \otimes M(\phi(C)) \bigotimes_{k Z_{\left.u^{\prime}(\phi())\right)}} \phi_{h_{C}}\left(g_{\eta}\right)
$$

with

$$
N(C):=\bigoplus_{i \in I_{C}(r, u)} X_{C}^{(i)} \quad \text { and } \quad M(\phi(C)):=\bigoplus_{i \in I_{C}(r, u)} X_{\phi(C)}^{\prime\left(\phi_{C}(i)\right)} .
$$

It suffices to show that $N \cong{ }_{\phi}^{\phi^{-1}} M_{\phi}^{\phi^{-1}}$ as $k G$-Hopf bimodules by the proof of Proposition 2.4.

Considering

$$
x \otimes(w) \rho_{C}^{(i)}\left(\left(\zeta_{\theta}\left(h_{C}^{-1}\right)\right)^{-1}\right) \bigotimes_{k Z_{u(C)}} g_{\theta}=x \otimes w \bigotimes_{k Z_{u(C)}} g_{\eta} h_{c},
$$

then $\psi: N \rightarrow M$ given by

$$
\psi\left(x \otimes(w) \rho_{C}^{(i)}\left(\left(\zeta_{\theta}\left(h_{C}^{-1}\right)\right)^{-1}\right) \bigotimes_{k Z_{u(C)}} g_{\theta}\right)=\phi(x) \otimes \xi_{C}^{(i)}(w) \bigotimes_{k Z_{u^{\prime}(\phi(C))}} \phi_{h_{C}}\left(g_{\eta}\right)
$$

for any $x, y \in G$ with $x^{-1} y=g_{\theta}^{-1} u(C) g_{\theta}$, and $i \in I_{C}(r, u), w \in X_{C}^{(i)}$, where $C \in \mathcal{K}_{r}(G)$ and

$$
g_{\theta} h_{C}^{-1}=\zeta_{\theta}\left(h_{C}^{-1}\right) g_{\eta}, \quad \text { with } \zeta_{\theta}\left(h_{C}^{-1}\right) \in Z_{u(C)} \text { and } \theta, \eta \in \Theta_{C},
$$

is a $k$-linear isomorphism. It is clear that $\psi$ is a homomorphism not only of $k G$ bicomodules from $N$ to ${ }^{\phi^{-1}} M^{\phi^{-1}}$ but also of left $k G$-modules from $N$ to ${ }_{\phi} M$. 
For any $h \in G$ and $w \in X_{C}^{(i)}$,

$$
\begin{aligned}
\psi((x & \left.\left.\otimes(w) \rho_{C}^{(i)}\left(\left(\zeta_{\theta}\left(h_{C}^{-1}\right)\right)^{-1}\right) \bigotimes_{k Z_{u(C)}} g_{\theta}\right) \cdot h\right) \\
& =\psi\left(\left(x h \otimes(w) \rho_{C}^{(i)}\left(\left(\zeta_{\theta}\left(h_{C}^{-1}\right)\right)^{-1} \zeta_{\theta}(h)\right) \bigotimes_{k Z_{u(C)}} g_{\theta^{\prime}}\right)\right. \\
& =\phi(x h) \otimes \xi_{C}^{(i)}\left((w) \rho_{C}^{(i)}\left(\left(\zeta_{\theta}\left(h_{C}^{-1}\right)\right)^{-1} \zeta_{\theta}(h) \zeta_{\theta^{\prime}}\left(h_{C}^{-1}\right)\right)\right) \bigotimes_{k Z_{u^{\prime}(\phi(C))}} \phi_{h_{C}}\left(g_{\eta^{\prime}}\right) \quad(\text { by }(2.7)) \\
& \left.=\phi(x) \phi(h) \otimes\left(\xi_{C}^{(i)}(w)\right) \rho_{\phi(C)}^{\left(\phi_{C}(i)\right)} \phi_{h_{C}}\left(\left(\zeta_{\theta}\left(h_{C}^{-1}\right)\right)^{-1} \zeta_{\theta}(h) \zeta_{\theta^{\prime}}\left(h_{C}^{-1}\right)\right)\right) \bigotimes_{k Z_{u^{\prime}(\phi(C))}} \phi_{h_{C}}\left(g_{\eta^{\prime}}\right)
\end{aligned}
$$

(by Definition 2.1)

and

$$
\begin{aligned}
\psi(x & \left.\otimes(w) \rho_{C}^{(i)}\left(\left(\zeta_{\theta}\left(h_{C}^{-1}\right)\right)^{-1}\right) \bigotimes_{k Z_{u(C)}} g_{\theta}\right) \cdot \phi(h) \\
& =\left(\phi(x) \otimes \xi_{C}^{(i)}(w) \bigotimes_{k Z_{u^{\prime}(\phi(C))}} \phi_{h_{C}}\left(g_{\eta}\right)\right) \cdot \phi(h) \\
& =\phi(x) \phi(h) \otimes\left(\xi_{C}^{(i)}(w)\right) \rho_{\phi(C)}^{\left(\phi_{C}(i)\right)} \phi_{h_{C}}\left(\zeta_{\eta}\left(h_{C} h h_{C}^{-1}\right)\right) \bigotimes_{k Z_{u^{\prime}(\phi(C))}} \phi_{h_{C}}\left(g_{\eta^{\prime}}\right) \quad(\text { by }(2.6)) \\
& =\phi(x) \phi(h) \otimes\left(\xi_{C}^{(i)}(w)\right) \rho_{\phi(C)}^{\left(\phi_{C}(i)\right)} \phi_{h_{C}}\left(\left(\zeta_{\theta}\left(h_{C}^{-1}\right)\right)^{-1} \zeta_{\theta}(h) \zeta_{\theta^{\prime}}\left(h_{C}^{-1}\right)\right) \bigotimes_{k Z_{u^{\prime}(\phi(C))}} \phi_{h_{C}}\left(g_{\eta^{\prime}}\right)
\end{aligned}
$$

(by (2.5)),

which shows that $\psi$ is a right $k G$-module homomorphism.

(ii) $\Rightarrow$ (i). Assume that there exist a Hopf algebra isomorphism $\phi: k G \rightarrow k G^{\prime}$ and a $k G$-Hopf bimodule isomorphism

$$
\psi:\left(k Q_{1}^{c}, G, r, \vec{\rho}, u\right) \rightarrow{ }_{\phi}^{\phi^{-1}}\left(k Q_{1}^{\prime c}, G^{\prime}, r^{\prime}, \vec{\rho}^{\prime}, u^{\prime}\right)_{\phi}^{\phi^{-1}} .
$$

Then $\phi: G \rightarrow G^{\prime}$ is a group isomorphism. Let $C \in \mathcal{K}(G)$. Then $\phi(u(C)), u^{\prime}(\phi(C)) \in$ $\phi(C) \in \mathcal{K}\left(G^{\prime}\right)$, and hence

$$
u^{\prime}(\phi(C))=\phi\left(h_{C}\right)^{-1} \phi(u(C)) \phi\left(h_{C}\right)=\phi\left(h_{C}^{-1} u(C) h_{C}\right)
$$

for some $h_{C} \in G$. Since $\psi$ is a $k G^{\prime}$-bicomodule isomorphism from ${ }^{\phi}\left(k Q_{1}^{c}, G, r, \vec{\rho}, u\right)^{\phi}$ to $\left(k Q_{1}^{\prime c}, G^{\prime}, r^{\prime}, \vec{\rho}^{\prime}, u^{\prime}\right)$ and

$$
\phi\left(h_{C}^{-1} u(C) h_{C}\right)=u^{\prime}(\phi(C)),
$$

by restriction we get a $k$-linear isomorphism

$$
\psi_{C}: h_{C}^{-1} u(C) h_{C}\left(k Q_{1}\right)^{1} \rightarrow{ }^{u^{\prime}(\phi(C))}\left(k Q_{1}^{\prime}\right)^{1}, \quad x \mapsto \psi(x) .
$$


We also have a $k$-linear isomorphism

$$
f_{C}:{ }^{u(C)}\left(k Q_{1}\right)^{1} \rightarrow{ }^{h_{C}^{-1} u(C) h_{C}}\left(k Q_{1}\right)^{1}, \quad x \mapsto h_{C}^{-1} \cdot x \cdot h_{C} .
$$

Since

$$
\phi\left(h_{C}^{-1} u(C) h_{C}\right)=u^{\prime}(\phi(C)) \quad \text { and } \quad h_{C}^{-1} Z_{u(C)} h_{C}=Z_{h_{C}^{-1} u(C) h_{C}},
$$

we have

$$
\phi\left(h_{C}^{-1} Z_{u(C)} h_{C}\right)=Z_{u^{\prime}(\phi(C))} .
$$

Hence $\phi_{h_{C}}$ is an algebra isomorphism from $k Z_{u(C)}$ to $k Z_{u^{\prime}(\phi(C))}$ sending $h$ to $\phi\left(h_{C}^{-1} h h_{C}\right)$. Using the hypothesis that $\psi$ is a $k G$-bimodule homomorphism from $\left(k Q_{1}^{c}, G, r, \vec{\rho}, u\right)$ to ${ }_{\phi}\left(k Q_{1}^{\prime c}, G^{\prime}, r^{\prime}, \vec{\rho}^{\prime}, u^{\prime}\right)_{\phi}$, we can easily check that the composition $\psi_{C} f_{C}$ is a right $k Z_{u(C)^{-}}$ module isomorphism from $\left(^{u(C)}\left(k Q_{1}\right)^{1}, \triangleleft\right)$ to $\left(\left({ }^{u^{\prime}(\phi(C))}\left(k Q_{1}^{\prime}\right)^{1}\right)_{\phi_{h_{C}}}, \triangleleft\right)$. Indeed, for any $z \in^{u(C)}\left(k Q_{1}\right)^{1}$,

$$
\begin{aligned}
\psi_{C} f_{C}(z) \triangleleft \phi_{h_{C}}(h) & =\psi_{C}\left(z \triangleleft h_{C}\right) \triangleleft \phi_{h_{C}}(h) \\
& =\psi_{C}\left(z \triangleleft h_{C} \phi_{h_{C}}(h)\right) \quad \text { (since } \psi \text { is a bimodule homomorphism) } \\
& =\psi_{C} f_{C}(z \triangleleft h) .
\end{aligned}
$$

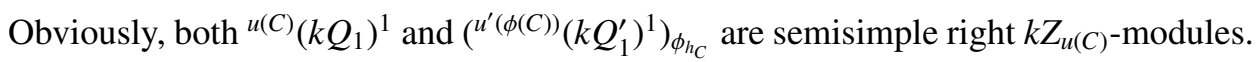
Assume

$$
{ }^{u(C)}\left(k Q_{1}\right)^{1} \cong \bigoplus_{i \in I_{C}(r, u)}\left(X_{C}^{(i)}, \rho_{C}^{(i)}\right)
$$

as right $k Z_{u(C)}$-modules and

$$
u^{\prime}(\phi(C))\left(k Q_{1}^{\prime}\right)^{1} \cong \bigoplus_{j \in I_{\phi(C)}\left(r^{\prime}, u\right)}\left(X_{\phi(C)}^{\prime(j)}, \rho_{\phi(C)}^{\prime(j)}\right)
$$

as right $k Z_{u^{\prime}(\phi(C))}$-modules, where $\left(X_{C}^{(i)}, \rho_{C}^{(i)}\right)$ is an irreducible right $k Z_{u(C)}$-module for any $i \in I_{C}(r, u)$ and $\left(X_{\phi(C)}^{\prime(j)}, \rho_{\phi(C)}^{\prime(j)}\right)$ is an irreducible right $k Z_{u^{\prime}(\phi(C))}$-module for any $j \in I_{\phi(C)}\left(r^{\prime}, u^{\prime}\right)$. Therefore, there exists a bijective map $\phi_{C}: I_{C}(r, u) \rightarrow I_{\phi(C)}\left(r^{\prime}, u^{\prime}\right)$ such that $\left(X_{C}^{(i)}, \rho_{C}^{(i)}\right) \cong\left(X_{\phi(C)}^{\prime\left(\phi_{C}(i)\right)}, \rho_{\phi(C)}^{\left(\phi_{C}(i)\right)} \phi_{h_{C}}\right)$ as right $k Z_{u(C)}$-modules for all $i \in I_{C}(r, u)$. It follows that $\operatorname{RSR}(G, r, \vec{\rho}, u) \cong \operatorname{RSR}\left(G^{\prime}, r^{\prime}, \overrightarrow{\rho^{\prime}}, u^{\prime}\right)$.

We have classified the quiver Hopf algebras by means of RSRs. In other words, ramification systems with irreducible representations uniquely determine their corresponding quiver Hopf algebras up to graded Hopf algebra isomorphisms.

Proposition 2.7. Let $\operatorname{RSR}(G, r, \vec{\rho}, u)$ and $\operatorname{RSR}\left(G, r, \overrightarrow{\rho^{\prime}}, u^{\prime}\right)$ be two $\operatorname{RSR}$ s. If $u^{\prime}(C)=$ $h_{C}^{-1} u(C) h_{C}$ and $\rho_{C}^{(i)}=\rho_{C}^{\prime(i)} \operatorname{ad}_{h_{C}}^{+}$with $I_{C}(r, u)=I_{C}\left(r, u^{\prime}\right)$ for any $C \in \mathcal{K}_{r}(G), i \in I_{C}(r, u)$, where $h_{C} \in G$ and $\operatorname{ad}_{h_{C}}^{+}(g)=h_{C}^{-1} g h_{C}$, then $\operatorname{RSR}(G, r, \vec{\rho}, u) \cong \operatorname{RSR}\left(G, r, \overrightarrow{\rho^{\prime}}, u^{\prime}\right)$.

Proof. Let $\phi=\mathrm{id}_{G}$ and $\phi_{C}=\mathrm{id}_{I_{C}(r, u)}$ for any $C \in \mathcal{K}_{r}(G)$. It is clear that $\operatorname{RSR}(G, r, \vec{\rho}, u) \cong \operatorname{RSR}\left(G, r, \overrightarrow{\rho^{\prime}}, u^{\prime}\right)$ 
REMARK 2.8. This proposition means that the choice of map $u$ does not affect the classification of RSRs. That is, if we fix a map $u_{0}$ from $\mathcal{K}(G)$ to $G$ with $u_{0}(C) \in C$ for any $C \in \mathcal{K}(G)$, then for any $\operatorname{RSR}(G, r, \vec{\rho}, u)$, there exists $\operatorname{RSR}\left(G, r, \overrightarrow{\rho^{\prime}}, u_{0}\right)$ such that $\operatorname{RSR}(G, r, \vec{\rho}, u) \cong \operatorname{RSR}\left(G, r, \overrightarrow{\rho^{\prime}}, u_{0}\right)$.

\section{Classification of pointed Hopf algebras of type one}

A graded Hopf algebra $A=\bigoplus_{n=0}^{\infty} A_{(n)}$ is said to be of Nichols type, if the diagram of $A$ is a Nichols algebra over $A_{(0)}$. (The definition of diagram was given in [1] and [20, Section 3.1].) Furthermore, if the coradical of $A$ is a group algebra, then $A$ is called a pointed Hopf algebra of Nichols type.

For an $\operatorname{RSR}(G, r, \vec{\rho}, u)$ and a $k G$-Hopf bimodule $\left(k Q_{1}^{c}, G, r, \vec{\rho}, u\right)$ with the module operations $\alpha^{-}$and $\alpha^{+}$, define a new left $k G$-action on $k Q_{1}$ by

$$
g \triangleright x:=g \cdot x \cdot g^{-1}, \quad g \in G, x \in k Q_{1},
$$

where $g \cdot x=\alpha^{-}(g \otimes x)$ and $x \cdot g=\alpha^{+}(x \otimes g)$ for any $g \in G$ and $x \in k Q_{1}$. With this left $k G$-action and the original left (arrow) $k G$-coaction $\delta^{-}, k Q_{1}$ is a YetterDrinfeld $k G$-module. Let $Q_{1}^{1}:=\left\{a \in Q_{1} \mid s(a)=1\right\}$, the set of all arrows with starting vertex 1 . It is clear that $k Q_{1}^{1}$ is a Yetter-Drinfeld $k G$-submodule of $k Q_{1}$, denoted by $\left(k Q_{1}^{1}, \operatorname{ad}(G, r, \vec{\rho}, u)\right)$

\section{LEMMA 3.1.}

(i) If $H$ is a Hopf algebra with bijective antipode and $\left(B, \alpha_{B}^{-}, \delta_{B}^{-}\right)$is a graded braided Hopf algebra in ${ }_{H}^{H} \mathcal{Y} D$ with $B_{(0)}=k 1_{B}$, then $\operatorname{diag}(B \# H)=B \# 1_{H} \cong B$ as graded braided Hopf algebras in ${ }_{H}^{H} \mathrm{yD}$.

(ii) $A$ is a pointed Hopf algebra of Nichols type if and only if $A$ is isomorphic to the biproduct $\mathfrak{B}(V) \# k G$ as graded Hopf algebras with the Nichols algebra $\mathfrak{B}(V)$ over the group algebra $k G, A_{(0)}=k G$ and $A_{(1)}=V \# k G$.

Proof. (i) Obviously, $\operatorname{diag}(B \# H)=B \otimes 1_{H}$. Define a map $\psi$ from $\operatorname{diag}(B \# H)$ to $B$ by sending $x \otimes 1_{H}$ to $x$ for any $x \in B$. It is easy to check that $\psi$ is a graded braided Hopf algebra isomorphism in ${ }_{H}^{H} \mathcal{Y} D$.

(ii) If $A$ is a pointed Hopf algebra of Nichols type, then $\operatorname{diag}(A)=\mathfrak{B}(V)$ is a Nichols algebra in ${ }_{k G}^{k G} \mathcal{Y} D$ and the coradical of $A$ is the group algebra $k G$. Therefore $A \cong$ $\mathfrak{B}(V) \# k G$ as graded Hopf algebras. By [1, Lemma 2.5], $A_{(0)}=k G$ and $A_{(1)}=V \# k G$.

Conversely, clearly, $\operatorname{diag}(\mathfrak{B}(V) \# k G)=(\mathfrak{B}(V) \# k G)^{\operatorname{cok} G}=\mathfrak{B}(V) \# 1 \cong \mathfrak{B}(V)$ by (i) and the coradical of $\mathfrak{B}(V) \# k G$ is $(\mathfrak{B}(V) \# k G)_{(0)}=k G$.

Lemma 3.2. If $H=k G$ is a group algebra and $M$ is an H-Hopf bimodule, then the pointed Hopf algebra $H[M]$ of type one is a Hopf algebra of Nichols type. In particular, a one-type-co-path Hopf algebra $k G\left[k Q_{1}^{c}, G, r, \vec{\rho}, u\right]$ is a pointed Hopf algebra of Nichols type and $\operatorname{diag}\left(k G\left[k Q_{1}^{c}, G, r, \vec{\rho}, u\right]\right)=\mathfrak{B}\left(k Q_{1}^{1}, \operatorname{ad}(G, r, \vec{\rho}, u)\right)$. 
Proof. By Proposition 2.4 there exists an $\operatorname{RSR}(G, \overrightarrow{r \rho}, u)$ such that $M \cong$ $\left(k Q_{1}^{c}, G, \overrightarrow{r \rho}, u\right)$ as $k G$-Hopf bimodules. Thus $k G[M] \cong k G\left[k Q_{1}^{c}\right]$ as graded Hopf algebras by [20, Lemma 1.6]. Therefore, it is enough to show that $k G\left[k Q_{1}^{c}\right]$ is of Nichols type. Let $A:=k G\left[k Q_{1}^{c}\right]$ and $R:=\operatorname{diag}\left(k G\left[k Q_{1}^{c}\right]\right)$. Obviously, $R_{(0)}=k$. We now show that $R_{(1)}=k Q_{1}^{1}$. Obviously, $k Q_{1}^{1} \subseteq R_{(1)}$. Let

$$
\alpha=\sum_{p=1}^{n} k_{p} b^{(p)} \in R_{(1)},
$$

where $b^{(p)}$ is an arrow from $x^{(p)}$ to $y^{(p)}$ with $0 \neq k_{p} \in k$, and $b^{(1)}, b^{(2)}, \ldots, b^{(n)}$ are different from each other. Therefore

$$
\sum_{p=1}^{n} k_{p} b^{(p)} \otimes 1=\sum_{p=1}^{n} k_{p} b^{(p)} \otimes x^{p},
$$

which implies that $x^{(p)}=1$ for $1 \leq p \leq n$. Thus $\alpha \in k Q_{1}^{1}$. We next show that $R$ is generated by $R_{(1)}$ as algebras. Let $\mu$ denote multiplication and let $B$ denote the algebra generated by $k Q_{1}^{1}$ in $k G\left[k Q_{1}^{c}\right]$. Obviously, $B \subseteq R$. It follows from the argument in [20, Section 3.1] that $\alpha^{+}:=\mu\left(\mathrm{id} \otimes \iota_{0}\right)$ is an algebraic isomorphism from $R \# k G$ to $k G\left[k Q_{1}^{c}\right]$. For any $x, y \in G$ and any arrow $a_{y, x}$ from $x$ to $y$,

$$
\begin{aligned}
a_{y, x} & =x \cdot a_{x^{-1} y, 1}=\mu\left(x \otimes a_{x^{-1} y, 1}\right)=\mu\left(\alpha^{+}(1 \# x) \otimes \alpha^{+}\left(a_{x^{-1} y, 1} \# 1\right)\right) \\
& =\alpha^{+}\left(\mu\left((1 \# x) \otimes\left(a_{x^{-1} y, 1} \# 1\right)\right)\right)=\alpha^{+}\left(x \triangleright a_{x^{-1} y, 1} \# x\right) \in \alpha^{+}(B \# k G) .
\end{aligned}
$$

Therefore $\alpha^{+}(B \# k G)=\alpha^{+}(R \# k G)$ and so $B=R$.

It is sufficient to show that $P(R)=k Q_{1}^{1}$, where $P(R)$ denotes the set of all primitive elements in $R$. For any $a \in Q_{1}^{1}$ with $\delta^{-}(a)=y$ and $\delta^{+}(a)=1$,

$$
\Delta_{R}(a)=(\omega \otimes \mathrm{id}) \Delta_{A}(a)=1 \otimes a+a \otimes 1
$$

(see [20, Section 3]), that is, $k Q_{1}^{1} \subseteq P(R)$, where

$$
\omega=\mu_{A}\left(\mathrm{id} \otimes \iota_{0} \pi_{0} S\right) \Delta_{A} .
$$

Conversely, we shall show that $P(R) \subseteq k Q_{1}^{1}$ by the following two steps. Obviously, $k G \cap P(R)=0$ and $P(R)$ is a graded subspace of $R$.

(i) Assume that $\alpha=a_{x_{n} x_{n-1}} a_{x_{n-1} x_{n-2}} \ldots a_{x_{1} x_{0}}$ is a path from vertex $x_{0}$, via arrows $a_{x_{1} x_{0}}, \ldots, a_{x_{n-1} x_{n-2}}, a_{x_{n} x_{n-1}}$, to vertex $x_{n}$. Then $\omega(\alpha)=\alpha \cdot x_{0}^{-1}$.

(ii) Let

$$
v=\sum_{p=1}^{m} k_{p} \alpha_{p} \in P(R),
$$

where

$$
\alpha_{p}=b_{x_{n} x_{n-1}}^{(p)} b_{x_{n-1} x_{n-2}}^{(p)} \ldots b_{x_{1} x_{0}}^{(p)}
$$


is a path with $n>1, k_{p} \in k$ for $p=1,2, \ldots, m$, and $b_{x_{j} x_{j-1}}^{(p)}$ is an arrow from vertex $x_{j-1}$ to vertex $x_{j}$ for $j=1,2, \ldots, n$. We shall show that $k_{p}=0$ for $p=1,2, \ldots, m$. Indeed,

$$
\begin{aligned}
\Delta_{R}(v) & =\sum_{p=1}^{m} \sum_{j=0}^{n} k_{p}\left(b_{x_{n} x_{n-1}}^{(p)} b_{x_{n-1} x_{n-2}}^{(p)} \ldots b_{x_{j+1} x_{j}}^{(p)}\right) \cdot\left(x_{j}^{(p)}\right)^{-1} \otimes b_{x_{j} x_{j-1}}^{(p)} b_{x_{j-1} x_{j-2}}^{(p)} \ldots b_{x_{1} x_{0}}^{(p)} \\
& =\sum_{p=1}^{m} k_{p}\left(\alpha_{p} \otimes 1+1 \otimes \alpha_{p}\right) .
\end{aligned}
$$

This implies that

$$
\sum_{p=1}^{m} k_{p}\left(b_{x_{n} x_{n-1}}^{(p)} b_{x_{n-1} x_{n-2}}^{(p)} \ldots b_{x_{j+1} x_{j}}^{(p)}\right) \cdot\left(x_{j}^{(p)}\right)^{-1} \otimes b_{x_{j} x_{j-1}}^{(p)} b_{x_{j-1} x_{j-2}}^{(p)} \ldots b_{x_{1} x_{0}}^{(p)}=0
$$

for $j=1,2, \ldots, n-1$, because of their length. For any $j$ with $1 \leq j \leq n-1$, assume that

$$
\left\{p \mid b_{x_{j} x_{j-1}}^{(p)} b_{x_{j-1} x_{j-2}}^{(p)} \ldots b_{x_{1} x_{0}}^{(p)}=b_{x_{j} x_{j-1}}^{(1)} b_{x_{j-1} x_{j-2}}^{(1)} \ldots b_{x_{1} x_{0}}^{(1)}\right\}=\left\{1,2, \ldots, m_{1}\right\}
$$

without loss of generality. Therefore, by (3.1),

$$
\sum_{p=1}^{m_{1}} k_{p}\left(b_{x_{n} x_{n-1}}^{(p)} b_{x_{n-1} x_{n-2}}^{(p)} \ldots b_{x_{j+1} x_{j}}^{(p)}\right) \cdot\left(x_{j}^{(p)}\right)^{-1} \otimes b_{x_{j} x_{j-1}}^{(p)} b_{x_{j-1} x_{j-2}}^{(p)} \ldots b_{x_{1} x_{0}}^{(p)}=0
$$

since

$$
b_{x_{j} x_{j-1}}^{(q)} b_{x_{j-1} x_{j-2}}^{(q)} \ldots b_{x_{1} x_{0}}^{(q)} \neq b_{x_{j} x_{j-1}}^{(1)} b_{x_{j-1} x_{j-2}}^{(1)} \ldots b_{x_{1} x_{0}}^{(1)} \quad \text { for } q=m_{1}+1, \ldots, m .
$$

This implies that

$$
\sum_{p=1}^{m_{1}} k_{p} b_{x_{n} x_{n-1}}^{(p)} b_{x_{n-1} x_{n-2}}^{(p)} \ldots b_{x_{j+1} x_{j}}^{(p)}=0 \quad \text { and } \quad k_{p}=0 \text { for } p=1,2, \ldots, m_{1} .
$$

Similarly, we can show that $k_{p}=0$ for $p=m_{1}+1, \ldots, m$.

By [5, Theorem 4.3.2], the category ${ }_{H}^{H} \mathcal{Y} D$ of Yetter-Drinfeld modules is equivalent to the category ${ }_{H}^{H} \mathcal{M}_{H}^{H}$ of $H$-Hopf bimodules, where $H$ is a Hopf algebra with bijective antipode. Let $T$ and $U$ be the two corresponding functors. For any $N \in_{H}^{H} \mathcal{Y} D$, according to [5, Proposition 4.2.1], $T(N):=N \rtimes H=N \otimes H$ as vector spaces, and the actions and coactions are given as follows: the left (co)actions are diagonal and right (co)actions are induced by $H$. Explicitly, $g \cdot(x \otimes h):=g \cdot x \otimes g h,(x \otimes h) \cdot g=$ $x \otimes h g, \delta_{N \rtimes H}^{-}(x \otimes h):=\sum_{x} x_{(-1)} h \otimes x_{(0)} \otimes h ; \delta_{N \rtimes H}^{+}(x \otimes h):=x \otimes h \otimes h$, where

$$
\delta_{N}^{-}(x)=\sum_{x} x_{(-1)} \otimes x_{(0)}, \quad x \in N, h, g \in H .
$$


For any $M \in_{H}^{H} \mathcal{M}_{H}^{H}$, according to [5, Equations (7) and (21)], $U(M)$ is the coinvariant of $M$ as a vector space, that is,

$$
U(M):=M^{\mathrm{co} H}:=\left\{x \in M \mid \delta_{N}^{+}(x)=x \otimes 1\right\} .
$$

The left action is left adjoint action and the left coaction is the restricted coaction of the original coaction of $M$. That is,

$$
\alpha_{U(M)}^{-}(h \otimes x)=h \triangleright_{\text {ad }} x:=\alpha_{M}^{+}\left(\alpha_{M}^{-}(h \otimes x) \otimes h^{-1}\right)=(h \cdot x) \cdot h^{-1}
$$

and $\delta_{U(M)}^{-}(x)=\delta_{M}^{-}(x)$ for any $h \in H, x \in U(M)$. In fact, $T U(M)=U(M) \rtimes H$ and $U T(N)=N \otimes 1_{H}$. Let $\lambda_{N}$ be the map from $N \otimes 1_{H}$ to $N$ sending $x \otimes 1_{H}$ to $x$ for any $x \in N$, and let $v_{M}$ be the map from $U(M) \rtimes H$ to $M$ sending $x \otimes h$ to $\alpha_{M}^{+}(x \otimes h)=x \cdot h$ for any $x \in U(M)$ and $h \in H$. Then $\lambda$ and $v$ are the natural isomorphisms from functor $U T$ to id and from functor $T U$ to id, respectively. Note that the inverse of $v_{M}$ is

$$
\left(\alpha_{M}^{+} \otimes \mathrm{id}\right)(\mathrm{id} \otimes S \otimes \mathrm{id})\left(\delta_{M}^{+} \otimes \mathrm{id}\right) \delta_{M}^{+} .
$$

REMARK 3.3. We have $U\left(k Q_{1}^{c}, G, r, \vec{\rho}, u\right)=\left(k Q_{1}^{1}, \operatorname{ad}(G, r, \vec{\rho}, u)\right)$ by the proof of Lemma 3.2.

Lemma 3.4. Assume that $\phi$ is a Hopf algebra isomorphism from $H$ to $H^{\prime}$. Let $N \in H_{H^{\prime}}^{H^{\prime}} \mathcal{Y} D$ and $M \in{ }_{H}^{H^{\prime}} \mathcal{M}_{H^{\prime}}^{H^{\prime}}$. Then

$$
T\left({ }_{\phi}^{-1} N\right) \cong{ }_{\phi}^{-1} T(N)_{\phi}^{\phi^{-1}} \text { in }{ }_{H}^{H} \mathcal{M}_{H}^{H} \text { and } U\left({ }_{\phi}^{\phi_{\phi}^{-1}} M_{\phi}^{\phi^{-1}}\right) \cong{ }_{\phi}^{\phi^{-1}} U(M) \text { in }{ }_{H}^{H} \mathcal{Y} D .
$$

Proof. The first isomorphism is given by sending $x \otimes h$ to $x \otimes \phi(h)$ for any $x \in N$, $h \in H$; the second one is identity.

\section{Proposition 3.5.}

(i) If $N$ is a Yetter-Drinfeld $k G$-module, then there exists an $\operatorname{RSR}(G, r, \vec{\rho}, u)$ such that $N \cong\left(k Q_{1}^{1}\right.$, ad $\left.(G, r, \vec{\rho}, u)\right)$ as Yetter-Drinfeld $k G$-modules.

(ii) If $\mathfrak{B}(N)$ is a Nichols algebra in ${ }_{k G}^{k G} \mathcal{Y} D$, then there exists an $\operatorname{RSR}(G, r, \vec{\rho}, u)$ such that $\mathfrak{B}(N) \cong \mathfrak{B}\left(k Q_{1}^{1}, \operatorname{ad}(G, r, \vec{\rho}, u)\right)$ as graded braided Hopf algebras in ${ }_{k G}^{k G} \mathcal{Y} D$.

Proof. (i) Since $T(N)$ is a $k G$-Hopf bimodule, it follows from Proposition 2.4 that there exists an $\operatorname{RSR}(G, r, \vec{\rho}, u)$ such that $T(N) \cong\left(k Q_{1}^{c}, G, r, \vec{\rho}, u\right)$ as $k G$-Hopf bimodules. Thus, $N \cong U T(N) \cong U\left(k Q_{1}^{c}, G, r, \vec{\rho}, u\right)=\left(k Q_{1}^{1}, \operatorname{ad}(G, r, \vec{\rho}, u)\right)$ as YetterDrinfeld $k G$-modules by [5, Equations (7) and (21)].

(ii) This follows from (i) and [2, Corollary 2.3].

Lemma 3.6. Assume that $\phi$ is a Hopf algebra isomorphism from $H$ to $H^{\prime}$. Let $R$ and $R^{\prime}$ be graded braided Hopf algebras in ${ }_{H}^{H} \mathcal{Y} D$ and ${ }_{H^{\prime}}^{H^{\prime}} \mathcal{Y} D$ with $R_{(0)}=k 1_{R}$ and $R_{(0)}^{\prime}=k 1_{R^{\prime}}$, respectively. If $R$ and ${ }_{\phi}^{\phi^{-1}} R^{\prime}$ are isomorphic as graded braided Hopf algebras in ${ }_{H}^{H} y D$, then biproducts $R \# H \cong R^{\prime} \# H^{\prime}$ as graded Hopf algebras. 
Proof. Let $\psi$ be a graded braided Hopf algebra isomorphism from $R$ to ${ }_{\phi}^{\phi^{-1}} R^{\prime}$ in ${ }_{H}^{H} \mathcal{Y} D$. Define a map $v$ from $R \# H$ to $R^{\prime} \# H^{\prime}$ by sending $x \otimes h$ to $\psi(x) \otimes \phi(h)$ for any $x \in R, h \in H$. It is easy to check that $v$ is an isomorphism of graded Hopf algebras.

THEOREM 3.7. If $A$ is a pointed Hopf algebra of Nichols type with coradical $k G, a$ group algebra, then there exists a unique $\operatorname{RSR}(G, r, \vec{\rho}, u)$, up to isomorphism, such that $A \cong k G\left[k Q_{1}^{c}, G, r, \vec{\rho}, u\right]$ as graded Hopf algebras.

Proof. By Lemma 3.1, $A \cong \mathfrak{B}(V) \# k G$ as graded Hopf algebras. By Proposition 3.5(ii), there exists a $\operatorname{RSR}(G, r, \vec{\rho}, u)$ such that $\mathfrak{B}(V) \cong \mathfrak{B}\left(k Q_{1}^{1}, \operatorname{ad}(G, r, \vec{\rho}, u)\right)$ as graded braided Hopf algebras in ${ }_{k G}^{k G} \boldsymbol{y}$. Thus

$$
\begin{aligned}
k G\left[k Q_{1}^{c}, G, r, \vec{\rho}, u\right] & \cong \mathfrak{B}\left(k Q_{1}^{1}, \operatorname{ad}(G, r, \vec{\rho}, u)\right) \# k G \quad(\text { by Lemma 3.2) } \\
& \cong \mathfrak{B}(V) \# k G \quad(\text { by Lemma 3.6 }) \\
& \cong A .
\end{aligned}
$$

The uniqueness follows from [20, Theorem 3].

Considering Theorem 3.7 and Lemma 3.2, we have that $A$ is a pointed Hopf algebra of Nichols type if and only if $A$ is a pointed Hopf algebra of type one.

\section{Classification of Nichols algebras}

Theorem 4.1. Let $(G, r, \vec{\rho}, u)$ and $\left(G^{\prime}, r^{\prime}, \overrightarrow{\rho^{\prime}}, u^{\prime}\right)$ be two RSRs. Then the following statements are equivalent.

(i) $\operatorname{RSR}(G, r, \vec{\rho}, u) \cong \operatorname{RSR}\left(G^{\prime}, r^{\prime}, \overrightarrow{\rho^{\prime}}, u^{\prime}\right)$.

(ii) There exists a Hopf algebra isomorphism $\phi: k G \rightarrow k G^{\prime}$ such that $\left(k Q_{1}^{1}, \operatorname{ad}(G, r\right.$, $\vec{\rho}, u)) \cong \phi_{\phi}^{-1}\left(k Q_{1}^{\prime 1}, \operatorname{ad}\left(G^{\prime}, r^{\prime}, \overrightarrow{\rho^{\prime}}, u^{\prime}\right)\right)$ as Yetter-Drinfeld $k G$-modules.

(iii) There is a Hopf algebra isomorphism $\phi: k G \rightarrow k G^{\prime}$ such that $\mathfrak{B}\left(k Q_{1}^{1}, \operatorname{ad}(G, r\right.$, $\vec{\rho}, u)) \cong{ }_{\phi}^{\phi^{-1}} \mathfrak{B}\left(k Q_{1}^{\prime 1}, \operatorname{ad}\left(G^{\prime}, r^{\prime}, \overrightarrow{\rho^{\prime}}, u^{\prime}\right)\right)$ as graded braided Hopf algebra in ${ }_{k G}^{k G} \mathcal{Y} D$.

Proof. (i) $\Rightarrow$ (ii). We have

$$
\phi_{\phi}^{-1}\left(k Q_{1}^{\prime}{ }^{1}, \operatorname{ad}\left(G^{\prime}, r^{\prime}, \overrightarrow{\rho^{\prime}}, u^{\prime}\right)\right)={ }_{\phi}^{\phi^{-1}} U\left(k Q_{1}^{\prime c}, G^{\prime}, r^{\prime}, \overrightarrow{\rho^{\prime}}, u^{\prime}\right)
$$

(by the remark before Lemma 3.4)

$$
\begin{aligned}
& \cong U\left(\phi_{\phi}^{\phi^{-1}}\left(k Q_{1}^{\prime c}, G^{\prime}, r^{\prime}, \overrightarrow{\rho^{\prime}}, u^{\prime}\right)_{\phi}^{\phi^{-1}}\right) \quad(\text { by Lemma 3.4 }) \\
& \cong U\left(\left(k Q_{1}^{c}, G, r, \vec{\rho}, u\right)\right) \quad(\text { by Theorem 2.6) } \\
& =\left(k Q_{1}^{1}, \operatorname{ad}(G, r, \vec{\rho}, u)\right) .
\end{aligned}
$$

(ii) $\Rightarrow$ (iii). By [20, Lemma 2.7],

$$
\begin{aligned}
\mathfrak{B}\left(k Q_{1}^{1}, \operatorname{ad}(G, r, \vec{\rho}, u)\right) & \cong \mathfrak{B}\left({ }_{\phi}^{\phi^{-1}}\left(k Q_{1}^{\prime 1}, \operatorname{ad}\left(G^{\prime}, r^{\prime}, \overrightarrow{\rho^{\prime}}, u^{\prime}\right)\right)\right) \\
& \cong{ }_{\phi}^{\phi^{-1}} \mathfrak{B}\left(k Q_{1}^{\prime}{ }^{1}, \operatorname{ad}\left(G^{\prime}, r^{\prime}, \overrightarrow{\rho^{\prime}}, u^{\prime}\right)\right)
\end{aligned}
$$

as graded braided Hopf algebras in ${ }_{k G}^{k G} \mathcal{Y} D$. 
(iii) $\Rightarrow$ (i). We have $\mathfrak{B}\left(k Q_{1}^{1}\right) \# k G \cong \mathfrak{B}\left(k Q_{1}^{\prime 1}\right) \# k G^{\prime}$ as graded Hopf algebras by Lemma 3.6. Thus $k G\left[k Q_{1}^{c}\right] \cong \mathfrak{B}\left(k Q_{1}^{1}\right) \# k G \cong \mathfrak{B}\left(k Q_{1}^{\prime 1}\right) \# k G^{\prime} \cong k G^{\prime}\left[k Q_{1}^{\prime c}\right]$ as graded Hopf algebras by Lemma 3.2. Now (i) follows from Theorem 2.6.

We have classified all Nichols algebras by means of RSRs. In other words, ramification systems with irreducible representations uniquely determine their corresponding Nichols algebras up to pull-push graded braided Hopf algebra isomorphisms.

\section{Classification of RSRs over symmetric groups}

Let $\mathrm{ad}_{h}^{-}$and $\mathrm{ad}_{h}^{+}$denote the left and right adjoint actions, respectively. That is, $\operatorname{ad}_{h}^{-}(x):=h x h^{-1}$ for any $x \in G$. Let $\gamma_{C}=\left|Z_{s}\right|$ when $s \in C \in \mathcal{K}(G)$. Aut $G$ and Inn $G$ denote the automorphism group and inner automorphism group of $G$, respectively.

Definition 5.1. Let $\operatorname{RSR}(G, r, \vec{\rho}, u)$ and $\operatorname{RSR}\left(G^{\prime}, r^{\prime}, \overrightarrow{\rho^{\prime}}, u^{\prime}\right)$ be two RSRs. If there exists a bijective map $\phi_{C}$ from $I_{C}(r, u)$ to $I_{C}\left(r^{\prime}, u^{\prime}\right)$ such that $\rho_{C}^{(i)} \cong \rho_{C}^{\prime\left(\phi_{C}(i)\right)}$ for any $i \in I_{C}(r, u)$ and $C \in \mathcal{K}_{r}(G)$ with $G=G^{\prime}, r=r^{\prime}$ and $u=u^{\prime}$, then $\operatorname{RSR}(G, r, \vec{\rho}, u)$ and $\operatorname{RSR}\left(G^{\prime}, r^{\prime}, \overrightarrow{\rho^{\prime}}, u^{\prime}\right)$ are said to be of the same type. Furthermore, letting

$$
\widehat{Z_{u(C)}}=\left\{\xi_{u(C)}^{(i)} \mid i=1,2, \ldots, \gamma_{C}\right\}
$$

and

$$
n_{C}^{(i)}:=\left|\left\{j \mid \rho_{C}^{(j)} \cong \xi_{u(C)}^{(i)}\right\}\right|
$$

for any $C \in \mathcal{K}_{r}(G)$ and $1 \leq i \leq \gamma_{u(C)}$, then $\left\{\left(n_{C}^{(1)}, n_{C}^{(2)}, \ldots, n_{C}^{\left(\gamma_{C}\right)}\right)\right\}_{C \in \mathcal{K}_{r}(G)}$ is called the type of $\operatorname{RSR}(G, r, \vec{\rho}, u)$.

Lemma 5.2. If Aut $G=\operatorname{Inn} G$, for example, $G=\mathbb{S}_{n}$ with $n \neq 6$, then $\operatorname{RSR}(G, r, \vec{\rho}, u)$ and $\operatorname{RSR}\left(G, r, \overrightarrow{\rho^{\prime}}, u\right)$ are isomorphic if and only if they have the same type.

Proof. By [18, Proposition 1.1(ii)], Aut $G=\operatorname{Inn} G$ when $G=\mathbb{S}_{n}$ with $n \neq 6$.

Let $\operatorname{RSR}\left(G^{\prime}, r^{\prime}, \overrightarrow{\rho^{\prime}}, u^{\prime}\right)$ denote $\operatorname{RSR}\left(G, r, \overrightarrow{\rho^{\prime}}, u\right)$ with $G=G^{\prime}, r=r^{\prime}$ and $u=u^{\prime}$ for convenience. If $\operatorname{RSR}(G, r, \vec{\rho}, u)$ and $\operatorname{RSR}\left(G, r, \overrightarrow{\rho^{\prime}}, u\right)$ have the same type, then there exists a bijective map $\phi_{C}$ from $I_{C}(r, u)$ to $I_{\phi(C)}\left(r^{\prime}, u^{\prime}\right)$ such that $\rho_{C}^{\prime\left(\phi_{C}(i)\right)} \cong \rho_{C}^{(i)}$ for any $i \in I_{C}(r, u), C \in \mathcal{K}_{r}(G)$. Therefore they are isomorphic.

Conversely, if $\operatorname{RSR}(G, r, \vec{\rho}, u)$ and $\operatorname{RSR}\left(G, r, \overrightarrow{\rho^{\prime}}, u\right)$ are isomorphic, then there exist a $\phi \in \operatorname{Aut}(G), h_{C} \in G$ and a bijective map $\phi_{C}: I_{C}(r, u) \rightarrow I_{\phi(C)}\left(r^{\prime}, u^{\prime}\right)$ such that

$$
u^{\prime}(\phi(C))=\phi \operatorname{ad}_{h_{C}}^{+}(u(C))
$$

and

$$
\rho_{\phi(C)}^{\prime\left(\phi_{C}(i)\right)} \phi \operatorname{ad}_{h_{C}}^{+} \cong \rho_{C}^{(i)}
$$


for any $C \in \mathcal{K}_{r}(G)$ and $i \in I_{C}(r, u)$. Since $\phi \operatorname{ad}_{h_{C}}^{+} \in \operatorname{Aut} G=\operatorname{Inn} G$, there exists a $g_{C} \in G$ such that $\phi \operatorname{ad}_{h_{C}}^{+}=\operatorname{ad}_{g_{C}}^{+}$. Therefore $u(C)=\operatorname{ad}_{g_{C}}^{+}(u(C))$ and $\rho_{C}^{\prime\left(\phi_{C}(i)\right)} \operatorname{ad}_{g_{C}}^{+} \cong \rho_{C}^{(i)}$. That is,

$$
g_{C} \in Z_{u(C)} \quad \text { and } \quad \chi_{C}^{\prime\left(\phi_{C}(i)\right)} \operatorname{ad}_{g_{C}}^{+}(h)=\chi_{C}^{\prime\left(\phi_{C}(i)\right)}\left(g_{C}^{-1} h g_{C}\right)=\chi_{C}^{\prime\left(\phi_{C}(i)\right)}(h)=\chi_{C}^{(i)}(h)
$$

for any $h \in Z_{u(C)}$, where $\chi_{C}^{\prime\left(\phi_{C}(i)\right)}$ and $\chi_{C}^{(i)}$ denote the characters of $\rho_{C}^{\prime\left(\phi_{C}(i)\right)}$ and $\rho_{C}^{(i)}$, respectively. Consequently, $\chi_{C}^{\prime\left(\phi_{C}(i)\right)}=\chi_{C}^{(i)}$ and $\rho_{C}^{\prime\left(\phi_{C}(i)\right)} \cong \rho_{C}^{(i)}$. This implies that $\operatorname{RSR}(G, r, \vec{\rho}, u)$ and $\operatorname{RSR}\left(G, r, \overrightarrow{\rho^{\prime}}, u\right)$ have the same type.

For a given ramification $r$ of $G$, let $\Omega(G, r)$ be the set of all RSRs of $\mathrm{G}$ with the ramification $r$, namely,

$$
\Omega(G, r):=\{(G, r, \vec{\rho}, u) \mid(G, r, \vec{\rho}, u) \text { is an RSR }\}
$$

Let $\mathcal{N}(G, r)$ be the number of isomorphism classes in $\Omega(G, r)$.

TheOREM 5.3. Given a group $G$ and a ramification $r$ of $G$, assume Aut $G=\operatorname{Inn} G$, for example, $G=\mathbb{S}_{n}$ with $n \neq 6$. Let $u_{0}$ be a fixed map from $\mathcal{K}(G) \rightarrow G$ with $u_{0}(C) \in C$ for any $C \in \mathcal{K}(G)$. Let $\bar{\Omega}\left(G, r, u_{0}\right)$ denote the set consisting of all elements with distinct type in $\left\{\left(G, r, \vec{\rho}, u_{0}\right) \mid\left(G, r, \vec{\rho}, u_{0}\right)\right.$ is an $\left.\operatorname{RSR}\right\}$. Then $\bar{\Omega}\left(G, r, u_{0}\right)$ becomes the representative system of isomorphic classes in $\Omega(G, r)$.

Proof. For any $\operatorname{RSR}(G, r, \vec{\rho}, u)$, there exists $\operatorname{RSR}\left(G, r, \overrightarrow{\rho^{\prime}}, u_{0}\right)$ such that $\operatorname{RSR}(G, r, \vec{\rho}, u)$ $\cong \operatorname{RSR}\left(G, r, \overrightarrow{\rho^{\prime}}, u_{0}\right)$ by Proposition 2.7. Using Lemma 5.2, we complete the proof.

Corollary 5.4. We have

$$
\mathcal{N}(G, r)=\prod_{C \in \mathcal{K}_{r}(G)} \tau_{C},
$$

where $\tau_{C}$ is the number of elements in the set

$$
\left\{\left(n_{1}, n_{2}, \ldots, n_{\gamma_{C}}\right) \in \mathbb{N}^{\gamma_{C}} \mid n_{1} \operatorname{deg} \xi_{1}+n_{2} \operatorname{deg} \xi_{2}+\cdots+n_{\gamma_{C}} \operatorname{deg} \xi_{\gamma_{C}}=r_{C}\right\}
$$

for any $C \in \mathcal{K}_{r}(G)$.

Remark 5.5. For a given finite Hopf quiver $(Q, G, r)$ over the symmetric group $G=\mathbb{S}_{n}$ with $n \neq 6$, every path algebra $T_{(k G)^{*}}\left(k Q_{1}^{a}\right)$ over the quiver $(Q, G, r)$ admits exactly $\mathcal{N}(G, r)$ nonisomorphic graded Hopf algebra structures; every path coalgebra $T_{k G}^{c}\left(k Q_{1}^{c}\right)$ over the quiver $(Q, G, r)$ admits exactly $\mathcal{N}(G, r)$ nonisomorphic graded Hopf algebra structures.

\section{Appendix A.}

We now consider the dual case of Theorem 4.1. If $Q$ is finite, then $\left(k Q_{1}^{a}, G, r, \vec{\rho}, u\right)$ is a $(k G)^{*}$-Hopf bimodule with comodule operations $\delta^{-}$and $\delta^{+}$. Define a new left 
$(k G)^{*}$-coaction on $k Q_{1}^{a}$ given by

$$
\delta_{\text {coad }}^{-}(x):=\sum_{x} x_{(-1)} S\left(x_{(0)(1)}\right) \otimes x_{(0)(0)}, \quad \text { for any } x \in k Q_{1}^{a},
$$

that is, adjoint coaction. With this left $(k G)^{*}$-coaction and the original left (arrow) $(k G)^{*}$-action $\alpha^{-}, k Q_{1}^{a}$ is a Yetter-Drinfeld $(k G)^{*}$-module. Let $k Q_{1}^{1 a}$ denote the subspace spanned by $Q_{1}^{1}$ in $k Q_{1}^{a}$. It is clear that $\xi_{k Q_{1}^{c}}\left(k Q_{1}^{1}\right)=\left(k Q_{1}^{1 a}\right)^{*}$, where $\xi_{k Q_{1}^{c}}$ was defined in [20, Lemma 1.7]. Thus $k Q_{1}^{1 a}$ is a Yetter-Drinfeld $(k G)^{*}$-submodule of $k Q_{1}^{a}$, denoted by $\left(k Q_{1}^{1 a}, \operatorname{coad}(G, r, \vec{\rho}, u)\right)$, which is isomorphic to the dual of $\left(k Q_{1}^{1}\right.$, ad $\left.(G, r, \vec{\rho}, u)\right)$ as Yetter-Drinfeld $(k G)^{*}$-modules.

Therefore we have the dual case of Theorem 4.1.

Proposition A.1. Let $(G, r, \vec{\rho}, u)$ and $\left(G^{\prime}, r^{\prime}, \overrightarrow{\rho^{\prime}}, u^{\prime}\right)$ be two RSRs. Then the following statements are equivalent.

(i) $\operatorname{RSR}(G, r, \vec{\rho}, u) \cong \operatorname{RSR}\left(G^{\prime}, r^{\prime}, \overrightarrow{\rho^{\prime}}, u^{\prime}\right)$.

(ii) There exists a Hopf algebra isomorphism $\phi:(k G)^{*} \rightarrow\left(k G^{\prime}\right)^{*}$ such that

$$
\left(k Q_{1}^{1 a}, \operatorname{coad}(G, r, \vec{\rho}, u)\right) \cong \phi_{\phi}^{-1}\left(k Q_{1}^{\prime 1 a}, \operatorname{coad}\left(G^{\prime}, r^{\prime}, \overrightarrow{\rho^{\prime}}, u^{\prime}\right)\right)
$$

as Yetter-Drinfeld $(k G)^{*}$-modules.

(iii) There is a Hopf algebra isomorphism $\phi:(k G)^{*} \rightarrow\left(k G^{\prime}\right)^{*}$ such that

$$
\mathfrak{B}\left(k Q_{1}^{1 a}, \operatorname{coad}(G, r, \vec{\rho}, u)\right) \cong{ }_{\phi}^{\phi^{-1}} \mathfrak{B}\left(k Q_{1}^{\prime 1 a}, \operatorname{coad}\left(G^{\prime}, r^{\prime}, \vec{\rho}^{\prime}, u^{\prime}\right)\right)
$$

as graded braided Hopf algebras in ${ }_{(k G)^{*}}^{(k G} \mathcal{Y} D$.

Proof. Obviously, (ii) and Theorem 4.1(ii) are equivalent; (iii) and Theorem 4.1(iii) are equivalent.

If $V=\bigoplus_{i=0}^{\infty} V_{(i)}$ is a graded vector space, and $\operatorname{dim} V_{(i)}<\infty$ for $0 \leq i \leq \infty$, then $V$ is said to be locally finite. In this case, set

$$
V^{g}=: \bigoplus_{i=0}^{\infty}\left(V_{(i)}\right)^{*} \subseteq V^{*},
$$

as in [13].

Lemma A.2. Assume that $H=\bigoplus_{n=0}^{\infty} H_{(n)}$ is a locally finite graded Hopf algebra.

(i) Then

$$
H^{g}=: \bigoplus_{n=0}^{\infty}\left(H_{(n)}\right)^{*} \subseteq H^{0}
$$

is a locally finite graded Hopf algebra.

(ii) If $G$ is a finite group and the coradical $H_{0}$ of $H$ is $(k G)^{*}$, then the coradical $\left(H^{g}\right)_{0}$ of $H^{g}$ satisfies $\left(H^{g}\right)_{0} \cong k G$. 
Proof. (i) See [16], [17, Lemma 3.1.11].

(ii) Let

$$
C_{n}=: \sum_{i=0}^{n}\left(H_{(i)}\right)^{*} .
$$

Then $H^{g}=\sum_{n=0}^{\infty} C_{n}$ is a filtered coalgebra. By [13, Proposition 11.1.1], $C_{0}=$ $\left((k G)^{*}\right)^{*} \cong k G$ contains the coradical $\left(H^{g}\right)_{0}$ of $H^{g}$. Consequently, $\left(H^{g}\right)_{0} \cong k G$.

Proposition A.3. Let $G$ be a finite group. Assume that $V$ is a finite-dimensional $(k G)^{*}$ YD module. Then:

(i) the coradical of $\left(\mathfrak{B}(V) \#(k G)^{*}\right)^{g}$ is isomorphic to $k G$;

(ii) $\left(\mathfrak{B}(V) \#(k G)^{*}\right)^{g}$ is finite-dimensional if and only if $\mathfrak{B}(V)$ is finite-dimensional.

Proof. Let $R=: \mathfrak{B}(V)$ so that the bosonisation (or biproduct)

$$
R \#(k G)^{*}=\bigoplus_{i}^{\infty}\left(R_{(i)} \#(k G)^{*}\right)
$$

of $R$ and $(k G)^{*}$ is a local finite graded Hopf algebra with coradical $(k G)^{*}$. Then $\left(R \#(k G)^{*}\right)^{g}=: \bigoplus_{i}^{\infty}\left(R_{(i)} \#(k G)^{*}\right)^{*}$ is a graded Hopf algebra with coradical $\left(\left(R \#(k G)^{*}\right)^{g}\right)_{0} \cong k G$ by Lemma A.2. This proves (i).

For (ii), if $\left(R \#(k G)^{*}\right)^{g}$ is finite-dimensional, then there exists a natural number $m$ such that $\left(R \#(k G)^{*}\right)^{g}=\bigoplus_{i}^{m}\left(R_{(i)} \#(k G)^{*}\right)^{*}$. Consequently, $R \#(k G)^{*}=\bigoplus_{i}^{m}\left(R_{(i)} \#(k G)^{*}\right)$ and $\mathfrak{B}(V)$ is finite-dimensional. The converse is obvious.

Remark A.4. This gives a method to decide whether a Hopf algebra with coradical $(k G)^{*}$ is finite-dimensional or not by means of pointed Hopf algebras.

\section{Acknowledgements}

We would like to thank Professor N. Andruskiewitsch for his help. The first and third authors were financially supported by the Australian Research Council, and the second author was supported by NSF of China (10771183) and Sino-German project (GZ310). The first author wishes to thank the Department of Mathematics, University of Queensland, for its hospitality.

\section{References}

[1] N. Andruskiewitsch and H. J. Schneider, 'Lifting of quantum linear spaces and pointed Hopf algebras of order $p^{3}$, J. Algebra 209 (1998), 645-691.

[2] N. Andruskiewitsch and H. J. Schneider, 'Pointed Hopf algebras', in: New Directions in Hopf Algebras, Mathematical Sciences Research Institute Publications, 43 (Cambridge University Press, Cambridge, 2002), pp. 1-68.

[3] N. Andruskiewitsch and H. J. Schneider, 'On the classification of finite-dimensional pointed Hopf algebras', Ann. of Math. (2) 171 (2010), 375-417.

[4] N. Andruskiewitsch and S. Zhang, 'On pointed Hopf algebras associated to some conjugacy classes in $S_{n}$ ’, Proc. Amer. Math. Soc. 135 (2007), 2723-2731. 
[5] Y. Bespalov and B. Drabant, 'Hopf (bi-)modules and crossed modules in braided monoidal categories', J. Pure Appl. Algebra 123 (1998), 105-129.

[6] C. Cibils and M. Rosso, 'Algebres des chemins quantiques', Adv. Math. 125 (1997), 171-199.

[7] C. Cibils and M. Rosso, 'Hopf quivers', J. Algebra 254 (2002), 241-251.

[8] R. Dijkgraaf, V. Pasquier and P. Roche, 'Quasi Hopf algebras, group cohomology and orbifold models', Nucl. Phys. B Proc. Suppl. 18 (1991), 60-72.

[9] M. R. Gaberdiel, 'An algebraic approach to logarithmic conformal field theory', Int. J. Mod. Phys. A 18 (2003), 4593-4638.

[10] I. Heckenberger, 'The Weyl groupoid of a Nichols algebra of diagonal type', Invent. Math. 164 (2006), 175-188.

[11] S Majid, 'Quasi-triangular Hopf algebras and Yang-Baxter equations', Int. J. Mod. Phys. A 5 (1990), 1-91.

[12] W. Nichols, 'Bialgebras of type one', Comm. Algebra 6 (1978), 1521-1552.

[13] M. E. Sweedler, Hopf Algebras (Benjamin, New York, 1969).

[14] F. Van Oystaeyen and P. Zhang, 'Quiver Hopf algebras', J. Algebra 280 (2004), 577-589.

[15] S. L. Woronowicz, 'Differential calculus on compact matrix pseudogroups (quantum groups)', Comm. Math. Phys. 122 (1989), 125-170.

[16] S. Zhang, Braided Hopf Algebras (Hunan Normal University Press, Chungsha, 1999).

[17] S. Zhang, 'The double bicrossproducts of braided Hopf algebras', Comm. Algebra 29 (2001), 31-66.

[18] S. Zhang, M. Wu and H. Wang, 'Classification of ramification systems for symmetric groups', Acta Math. Sin. 51 (2008), 253-264.

[19] S. Zhang and Y.-Z. Zhang, 'Structures and representations of generalized path algebras', Algebr. Represent. Theor. 10 (2007), 117-134.

[20] S. Zhang, Y.-Z. Zhang and H. X. Chen, 'Classification of PM quiver Hopf algebras', J. Algebra Appl. 6 (2007), 919-950.

SHOUCHUAN ZHANG, Department of Mathematics, Hunan University, Changsha 410082, PR China

and

School of Mathematics and Physics, The University of Queensland, Brisbane 4072, Australia

HUI-XIANG CHEN, Department of Mathematics, Yangzhou University, Yangzhou 225002, PR China

YAO-ZHONG ZHANG, School of Mathematics and Physics, The University of Queensland, Brisbane 4072, Australia e-mail: yzz@maths.uq.edu.au 